\title{
Information Metamaterials: bridging the physical world and digital world
}

\author{
Qian Ma and Tie Jun Cui
}

\footnotetext{
*Correspondence: tjcui@seu.edu.cn State Key Laboratory of Millimeter Wave, Southeast University, Nanjing 210096, China
}

\begin{abstract}
Over the past 5 years, digital coding and programmable metamaterials have been developed rapidly since their first exhibition in 2014. The iconic feature of the digital coding metamaterial is using digital codes like " 0 " and " 1 " to represent the distinct electromagnetic (EM) responses. This seemingly trivial progress has successfully reform the design theory from the effective medium to coding patterns, bridging the physical world and digital information world. More interestingly, beyond the simple coding on the parameters or patterns, the digital coding metamaterials are more intend to introduce the concept of direct interactions and operations of digital information within EM fields, to realize information processing, transmission or recognition. To accurately exhibit the informational specialties, we classify the coding metamaterials, digital metamaterials and programmable metamaterials, as well as other information-operating metamaterials, as information metamaterials. In this review article, we firstly introduce the digital coding concept, working mechanism, and related design methods. Then, three important theories including the scattering pattern calculation, convolution operation, and entropy of digital coding metamaterials, are discussed in details. Finally we introduce several system-level works based on the information metamaterials, such as the new-architecture wireless communication systems and reprogrammable imaging systems, to show the powerful manipulation capabilities of information metamaterials. As the next generation of information metamaterials, two proofof-concept smart metamaterials and their advanced architectures are discussed. In the summary, the development track of information metamaterials and future trends are presented.
\end{abstract}

Keywords: Information metamaterials, Reprogrammable metamaterials, Space-time-coding metamaterials, Programmable imager, Smart metamaterials

\section{Introduction}

Metamaterials are artificial materials, which are usually composed of periodic or quasiperiodic structures with subwavelength dimensions. Here, the affix "meta" is referred from the Greek word " $\mu \varepsilon t \alpha$ ", to represent the artificial materials with unique properties that do not exist or are difficult to realize in nature. The original idea of metamaterial dates back to 1968, Veselago first proposed the concept of "left-handed material or negative-index material" [1]. However, due to the lack of experimental verification, metamaterials have not been put into reality until 20 years ago, when Pendry, Smith and co-workers presented the negative-index metamaterials and successfully realized the metamaterials in microwave frequencies [2-4]. This opened a long-term research

(c) The Author(s). 2020 Open Access This article is distributed under the terms of the Creative Commons Attribution 4.0 International License (http://creativecommons.org/licenses/by/4.0/), which permits unrestricted use, distribution, and reproduction in any medium, provided you give appropriate credit to the original author(s) and the source, provide a link to the Creative Commons license, and indicate if changes were made. 
boom from microwave to optical domains. One significant phenomenon realized by metamaterials is double-negative materials, or negative-index materials [2-8]. Another popular characteristic is near-zero refractive index [9-11], further extending the index range from negative to zero and positive. Benefited from the above peculiar capabilities, metamaterials have brought fascinating applications like invisibility cloaking [12-17], EM black holes [18, 19], perfect lens [3], perfect imaging [20, 21], and special lenses [15, 22-27].

However, metamaterials in three-dimensional structures still face high processing difficulty, as well as high resonant losses, which impeded the further development and application of metamaterials. To overcome these drawbacks, the two-dimensional version of metamaterials, metasurfaces (or metafilms) were proposed [28] in 2009. Different from the bulk metamaterials, metasurfaces are almost planar structures with deep subwavelength thickness, which can be implemented by the printed circuit board technology in most ranges of microwave. Such low profile reduces not only the fabrication complexity but also the cost apparently. Besides, ultrathin profile provides lower insertion loss and more potential applications. Most importantly, metasurfaces with specific structures are able to interact in the electromagnetic (EM) wave fields, and thus generating the mutational phase and amplitude in ultrathin surface. Owing to the above merits, metasurfaces have received great attention on the realization of many functional devices, including perfect absorbers [29-33], polarization converters [34-37], vector-beam generator [38-41], holograms [42-44], and diverse EM wave manipulators [45-48]. The operating frequency of metasurfaces has been extended from microwave $[35,36]$ to terahertz wave $[46,47$, 49], visible light [29, 30], and even for acoustic wave [49-54]. In addition, metasurfaces in flexible forms like polydimethylsiloxane [49] and flexible printed circuit [55] have been realized as potential wearable applications.

Among suc multitudinous researches, digital coding and programmable metamaterials have become an important direction, since the initial idea was introduced by Cui et al. $[56,57]$. Inspired by the binary expression of digital circuits, the coding metamaterials firstly offer a perspective from digital world to design metamaterials. By encoding the phase responses of meta-atom as " 0 " and "1", the EM parameter is briefly connected with the digital expression [56]. Consequently, the conventional metasurface pattern configuration is totally digitized into coding pattern, which has introduced diverse ideas from information science, to simplify the design process and create more functionalities. One important theory is convolution method based on digital coding metamaterials [58], which have established a flexible operation between the coding patterns and far-field scattering patterns. Another basic theory is the information entropy for digital coding metamaterials [59], providing an estimation of the information carried by the scattering beams. Moreover, the additional theorem has presented the transform between low-bit and high-bit coding [60]. In addition to the digital coding on the phase responses, the concept is applicative to the other EM parameters like amplitude [61-63], polarization [64-66], and even the orthogonal parameters like orbital angular momentum (OAM) modes [64, 67-69].

The coding modulation on the EM scattering fields is not limited in the reflected fields [58-62, 64], but also is applied on transmitted fields [63, 70-75], and even on the acoustic fields [51]. To explore the coding modulations on more dimensions, programmable metamaterials with active components have been proposed [56]. 
Different from the tunable and reconfigurable metamaterials, the programmable metamaterials yield a more powerful ability on EM wave manipulations and more functionalities controlled in real time. For 1-bit phase-programmable metasurfaces [56, 76], abundant single- or multi-beam fields can be obtained with pre-designed specific coding patterns. Crucially, the programmable trait provides a new dimension in time domain, bringing space-time coding applications [77-80]. Harmonic beam steering is achieved with the time-varying coding patterns of information metamaterials [77, 78]. Such digital coding methods are logically developed into digital communication systems, and two communication systems have been realized based on the programmable metasurfaces $[79,80]$, from the spatial-beam coding and harmonic-frequency coding directions, proposed by Cui and Zhao et al., respectively [79, 80]. Another popular application for the programmable metamaterials is imaging [81-83]. With reprogrammable metasurface patterns, imaging information is no longer fixed but editable as desired [81]. In the frame of information metamaterials, digital machine-learning algorithm has been easily implemented on the reprogrammable metamaterials for dynamical imaging [82]. Corresponding to the phase-coding, reprogrammable functionalities for amplitude modulations and non-reciprocal transmissions are also realized [84, 85]. For information devices, intelligence is an inevitable trend, and this is the same for the information metamaterials. For the aforementioned information metamaterials, no matter passive or active, the human instructions are necessary. To break through this limitation, the concept of smart metamaterials is presented [86], with a new architecture for independent decision-making. Besides, by integrating artificial intelligence, the smart imager and recognizer are realized [87].

Regardless of the early passive coding metamaterials or the later active programmable metamaterials, their digital forms enable the analysis, design and application from an informational perspective. Consequently, we would like to summarize a definition for information metamaterial with three features: (1) informational design: the metamaterial configuration can be designed with the digital characterization method or coding method; (2) informational analysis: the metamaterial performance can be analyzed from information or digital perspective; (3) informational processing: the metamaterials are able to process information operations or interactions in the physical wave fields. It should be mentioned that the above features are not necessarily all matched. We will further illustrate these features detailedly in the following sections.

This paper aims to review the development of information metamaterials, from the early original concepts to various powerful systems so far. In recent 6 years, the information metamaterials have gradually grown up to an important subject branch of metamaterials, including abundant functionalities, principles, and application systems. Different from the previous review papers on coding metmaterials, here we focus on the informational representation, interaction and operations based on the coding and programmable metasurfaces. We start the introduction from the basic concept and design ideas of digital coding metamaterials in Section 2, as well as their multitudinous functionalities on EM wave manipulations. In Section 3, we introduce several important principles and operations of the information metamaterials, such as the convolution theorem, information entropy and so on, which combined the conventional physical properties and digital states and established the theory system of information metamaterials. From Sections 4 to 6, we introduce three kinds of significant systems of 
information metamaterials, to exhibit the application directions in wireless communication and imaging. In Section 4, we discuss the space-time systems based on reprogrammable metamaterials, including three representative works on communications and EM-wave manipulators. In Section 5, we focus on the reprogrammable imaging systems using the information metamaterials, showing the performance on the metasurface hologram and real-time imaging. In Section 6, as the important developing directions of information metamaterials, two smart systems are presented, to respectively realize intelligent EM-wave manipulator, imager and recognizer. Finally, we summarize the development track and achievements of the information metamaterials, as well as the discussion on the future research spots.

\section{Concept and design of information metamaterials}

The most iconic character of information metamaterials is digitizing and discretization on meta-element, which produces a totally digital pattern on metamaterial configuration, namely coding patterns. This method offers us a new perspective to design metamaterial with multifarious functions. In this section, we start from the initial idea in the first presentation of coding metamaterial, to introduce the basic concept and design strategy, as well as various functionalities realized by the information metamaterial.

\section{The concept and design of the coding and programmable metamaterials}

The development of information metamaterials starts from the coding metamaterials proposed by Cui et al. [56]. The initial idea of coding metamaterials aims to design the metamaterials structure pattern within digital way, in which the design process is totally represented with digital coding pattern, as shown in Fig. 1a. To combine the digital coding pattern and EM wave fields, the first work we need is encoding the EM phase responses of unit-cell with "0/1" digits. For example, as shown in Fig. 1b, a coding element with patch structure is proposed, with the substrate of F4B (a dielectric constant of 2.65 and loss tangent of 0.001). In Fig. 1b, the reflected phase of the element is given from 7 to $14 \mathrm{GHz}$, where the phase difference is marked in the red dotted line. The reflected phase responses of the coding elements $0^{\circ}$ and $180^{\circ}$ are encoded into digits "0" and " 1 ". Therefore, for a metasurface with $8 * 8$ elements, the metasurface configuration is totally converted into a digital pattern as presented in Fig. 1a. In this coding method, reconsidering the previous metamaterials configuration, the design process is simplified into the "0/1" coding pattern design. In Fig. 1c and d, two coding patterns are provided with distinct scattering fields. Different from the conventional metamaterials, in the coding metamaterials, the functionality of the reflected- or transmissionfields can be edited by such digital patterns. The specific reflected fields are exhibited in Fig. 1e, where single-, dual- and quad-beam scattering fields are realized. Most importantly, the coding metamaterials offer us an information viewpoint to consider the EM scattering fields, implying that the EM fields are able to be expressed in digital way.

For passive structures, the EM responses are generously fixed, thereby their functions are also unalterable. To further extend the flexibility of the coding metamaterials, a programmable coding element, as well as a programmable metasurface, are presented [56], as shown in Fig. 2a and b. The metasurface element is composed of a single-layer substrate and two metal structure layers (the top and bottom), as illustrated in Fig. 2c. 

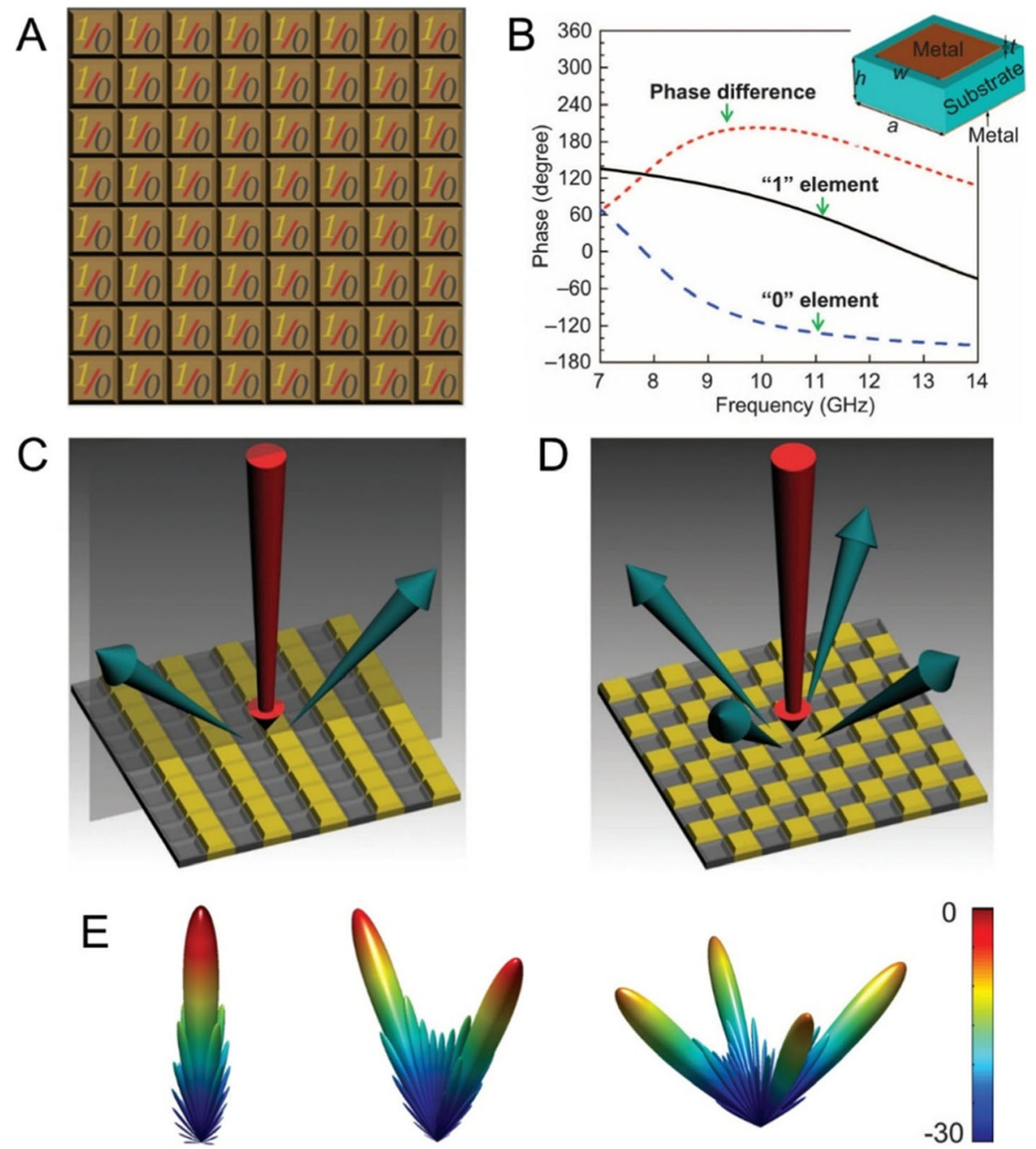

Fig. 1 The 1-bit digital coding metamaterials. a The 1-bit digital coding pattern of metamaterials. (from Fig. 1 of Ref. [56]). b A typical patch coding element with " 0 " and " $180^{\circ}$ " phase responses, encoded as bits " 0 " and "1". (from Fig. 1 of Ref. [56]). $\mathbf{c}$ and $\mathbf{d}$ The two designed coding patterns. (from Fig. 1 of Ref. [56]). e The simulated scattering fields for different patterns. (from Fig. 2 of Ref. [56]). Reproduced with permission from Ref. [56] under a Creative Commons Attribution-NonCommercial-ShareAlike 3.0 Unported License. Copyright 2014, Springer Nature

A PIN diode (Skyworks, SMP-1320) is embedded on the top metal structure of the coding element, to change the electric structure. To implement the bias voltage for PIN diodes, the metal structure on the top surface is connected to background, which also acts the direct current (DC) electrodes, with the via holes. Such alteration results in different phase responses, which have $180^{\circ}$ difference as shown in Fig. $2 \mathrm{~d}$. It is obvious that the phase difference at $8.6 \mathrm{GHz}$ is $180^{\circ}$, which can be exactly encoded as digital codes " 0 " and " 1 ". The " $0 / 1$ " states can be modulated with bias voltages from the field-programmable gate array (FPGA), as depicted in Fig. 2e. By arranging different coding sequences like "000000", "111,111", "010101", and "001011", various scattering patterns can be obtained, as shown in Fig. 1e. Besides, except for the beam-deflection and multi-beam fields, other functions like radar-cross-section (RCS) reduction is achieved [56]. 


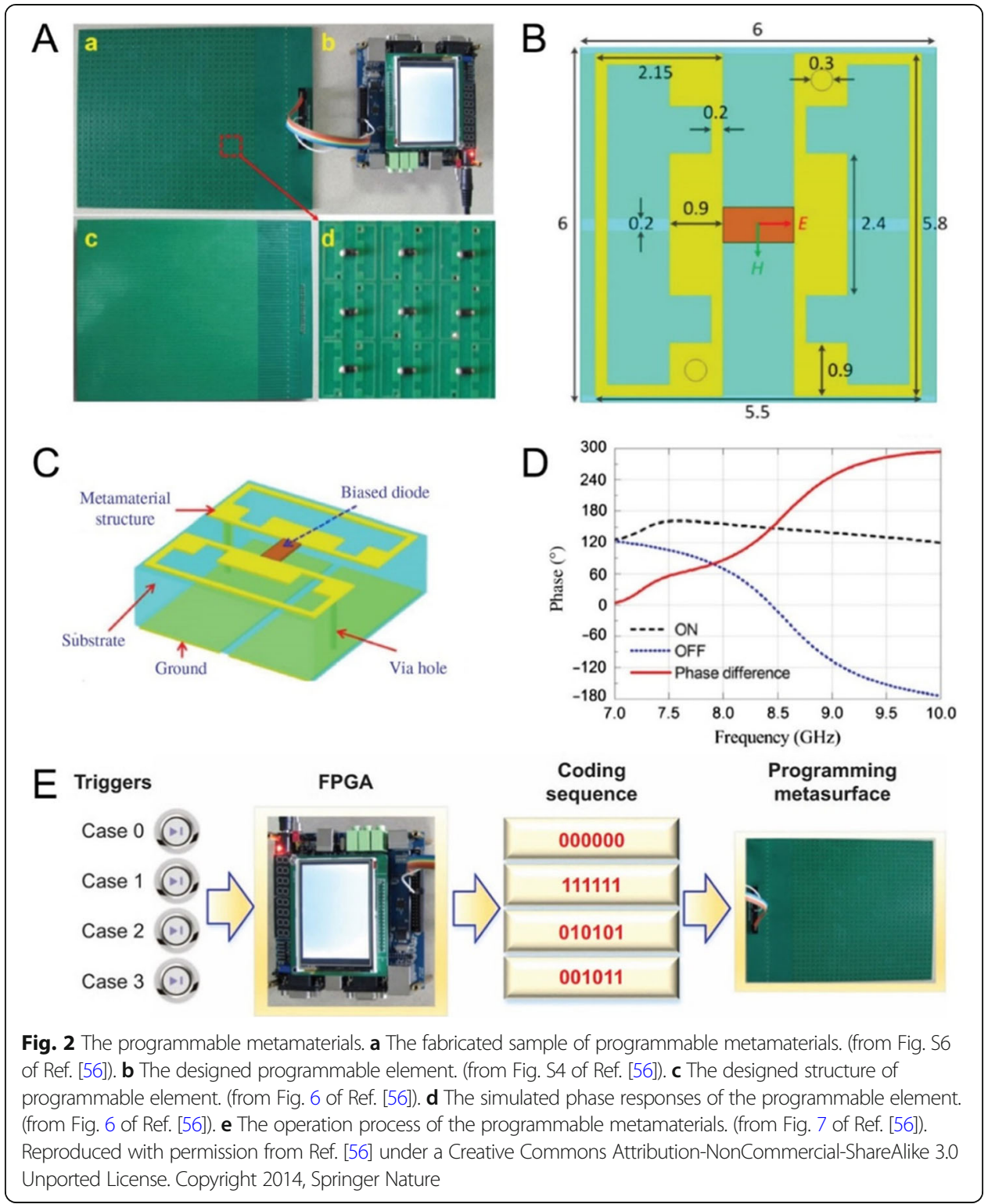

In the design method of information metamaterials, we summarize three key steps as following: (1) encode EM parameters; (2) design coding patterns; (3) simulations and experiments. For the first step, we usually define phase responses as the binary codes "0" and "1" [56, 76]. Since the generalized Snell' law was proposed [91], the phase manipulation has become very popular due to its varied compelling functions [56, 58-60, 62-65]. Actually, not only the phase responses can be encoded, but also other factors of EM fields are available for arrangement. From the perspective of information metamaterials, we are more concerned with the information processing methods of metamaterials. In other words, the EM field parameters (phase, amplitude, polarization, etc.), like the numbers in the field of metamaterials, deal with the EM problems in an informational way. For instance, orthogonal factors like polarization and OAM modes are encoded to express more information bits [64], offering extra channel capacities. Moreover, special transmission states like non-reciprocal transmission are encoded as well 
$[84,85]$. Secondly, with available coding bits, we arrange the coding patterns of metasurfaces to obtain the specific functions. Taking phase-coding metamaterials as an example, the phase pattern primarily determines the scattering directions of a reflective or transmissive metasurface, according to the generalized Snell's law [91]. By applying basic scattering-pattern calculation and the convolution operations, various scattering patterns are obtained. For more customized design, a fast pattern algorithm is proposed recently [86], for arbitrary scattering direction in a single- or multi-beam field. Finally, the simulations and experiments should be performed for verifications.

\section{Various functionalities realized by information metamaterials}

Thanks to the outstanding manipulation capacity and simple configuration, information metamaterials have achieved luxuriant utilities from microwave to optics [56, 88, 90]. In Fig. 3, we list several typical works of information metamaterials, to exhibit their excellent performance on tailoring the EM field. Figure 3a presents a $\mathrm{THz}$ coding metamaterial for RCS reduction [88]. A coding element is presented based on a Minkowski closed-loop with two resonances at 0.8 and $1.6 \mathrm{THz}$, which helps smooth the phase response in the frequency domain. Hence, by applying the appropriate coding patterns, a broadband low reflection coding metasurface is realized. Figure $3 b$ exhibits a new modulation mechanism for information metamaterials [89], by using photodiodes and varactors. The programmable element integrated with varactors generates multiple phase states for reflected wave. To select these states, the light-controlled photodiodes are employed to dominate these varactors. As a result, the metasurface pattern is able to be totally modulated by visible light. Another popular topic of metasurfaces is the design of anisotropic functions [90, 92]. In Fig. 3c and d, an anisotropic coding metasurface is realized in terahertz [90], as well as a coding unit for dual-polarizations. The anisotropic design usually means multi-functions, since two or more degrees of freedom are utilized. In this work, the authors deployed different sizes in two orthogonal directions ( $\mathrm{x}$ - and $\mathrm{y}$-axis) in one element, and achieved total 16 units (2-bits in two polarizations). Under the illumination of different-polarization incidences, the metasurface scatters the energy into opposite angles. Also, as we mentioned above, non-reciprocal phenomena were also realized recently with information metamaterials [84, 85]. In Fig. 3e, a nonreciprocity-reprogrammable metasurface is proposed [84]. With the integration of adjustable amplifying transistors, the transmission of the metasurface is controlled by the supplying voltage, which is further controlled by FPGA. Thus, four distinct transmission states are successfully actualized and encoded into digital codes. In addition, the vector beams are realized on the metasurfaces [64, 93, 94]. In Fig. 3f, we show a vector beam modulator to encode information on the orthogonal polarization bit and OAM mode bit [64]. A special coding unit is applied in this work for independent control of polarization and reflection-phase, due to the orthogonality between $x-$, $y$-polarization and OAM modes. Comparing to the previous phase coding, such coding methods provide extra information bits, having higher channel capacity.

\section{Principle of information metamaterials}

After the coding process, the underlying purpose of information metamaterials is to perform digital interactions and operations directly based on the EM fields. For this 

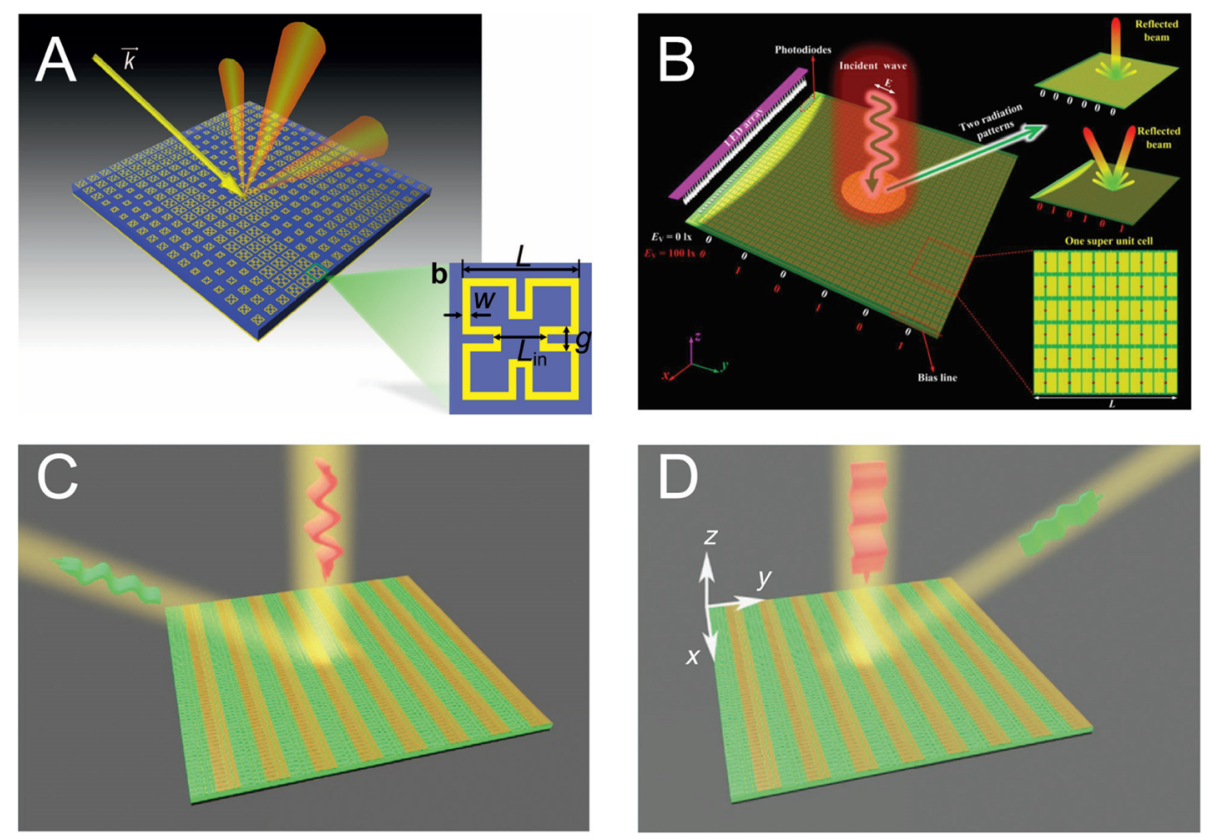

$E$

$\mathrm{F}$
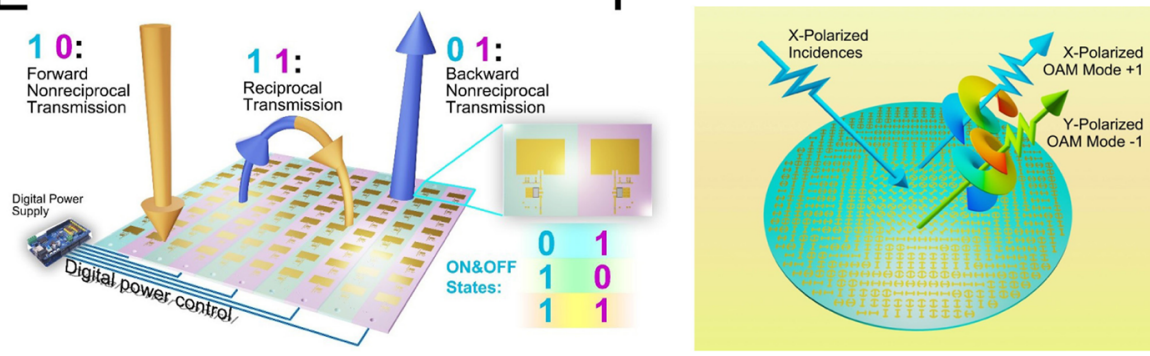

Fig. 3 The various functions realized by information metamaterials. a A broadband THz coding metasurface for RCS reduction. (from Fig. 1 of Ref. [88]). Reproduced with permission from Ref. [88] under a Creative Commons Attribution-NonCommercial-NoDerivs 4.0 International License. Copyright 2015, Springer Nature. b A light-controlled programmable metasurface. (from Fig. 1 of Ref. [89]). Reproduced with permission from Ref. [89] under the terms of the Creative Commons Attribution License. Copyright 2018, WILEY-VCH Verlag $\mathrm{GmbH} \&$ Co. KGaA, Weinheim. $\mathbf{c}$ and $\mathbf{d}$ The anisotropic coding metasurface in THz. (from Fig. 1 of Ref. [90]). Reproduced with permission from Ref. [90] under a Creative Commons Attribution-NonCommercial-NoDerivs 4.0 International License. Copyright 2016, Springer Nature. e A nonreciprocity-programmable metasurface. (from Fig. 1 of Ref. [84]). Reproduced with permission from Ref. [84]. Copyright 2019, WILEY-VCH Verlag GmbH \& Co. KGaA, Weinheim. $\mathbf{f}$ A combination coding metasurface on orthogonal polarizations and OAM modes. (from Fig. 1 of Ref. [64]). Reproduced with permission from Ref. [64]. Copyright 2017, WILEY-VCH Verlag GmbH \& Co. KGaA, Weinheim

purpose, several designs are proposed to establish the theoretical system. In this section, we mainly introduce three theories: the scattering pattern calculation, the convolution operation, and the information entropy, which occupy an important position in this theoretical framework of information metamaterials.

\section{The scattering pattern of information metamaterials}

For information metamaterials, the scattered-field calculation is the basis for the further functionality design. In Ref. [56, 76], the scattering pattern calculation for phase-coding metamaterials is elaborately illustrated. In this section, we will introduce this process 
briefly. In a generous situation, the information metamaterials are usually excited by a plane wave, as shown in Fig. 4a. For an $\mathrm{M}^{*} \mathrm{~N}$ metasurface, the functions of the scattering field in a spherical coordinate system can be written as [76],

$$
\mathrm{F}(\theta, \phi)=\sum_{m}^{M} \sum_{n}^{N} \begin{gathered}
\exp \left\{j \left[\phi_{m, n}+k_{0} D_{x}\left(m-\frac{1}{2}\right) \sin \theta \cos \phi+\right.\right. \\
\left.\left.k_{0} D_{y}\left(n-\frac{1}{2}\right) \sin \theta \cos \phi\right]\right\}
\end{gathered}
$$

where $\phi_{m, n}$ is the phase response of the element $(\mathrm{m}, \mathrm{n}), D_{x}$ and $D_{y}$ are the period length along $\mathrm{x}$ - and $\mathrm{y}$-axis. Once the coding pattern is determined, $\phi_{m, n}$ is certain, so the whole scattering field is calculated. In Fig. $4 \mathrm{~b}$ and c, two typical chess-board patterns are provided, in which the blue and yellow cells represent " 0 " and " 1 " codes, with $0^{\circ}$ and $180^{\circ}$ phase responses. The related scattering fields are shown in Fig. $4 \mathrm{~d}$ and e, where we can clearly observe the difference in the scattering directions of the four beams when the coding period varies.

\section{The convolution theorem of information metamaterials}

The above principle offers us a method to analyze a certain coding pattern. However, in most cases, the main demand is designing the pattern for a specific application, such as beam deflection. For that purpose, the convolution theorem of the information metamaterials is proposed [58]. Here, we use a 2-bit metamaterial as a proof of principle to introduce this operation, where the greyscales represent the codes " 0 ", "1", "2", and " 3 ". As shown in Fig. 5a-c, an addition operation on the coding patterns is presented, following the rules for quaternary numbers, but without carry-bit. Such a pattern operation corresponds to the reflected-beam rotation, as depicted in Fig. 5d-f. As we all known, a spectrum is moved by an impulse function in the frequency domain.

$$
\boldsymbol{f}(\boldsymbol{t}) \cdot \boldsymbol{e}^{j \omega_{0} t} \stackrel{F F T}{\Leftrightarrow} \mathbf{f}(\boldsymbol{\omega}) * \boldsymbol{\delta}\left(\boldsymbol{\omega}-\omega_{0}\right)=\boldsymbol{f}\left(\boldsymbol{\omega}-\boldsymbol{\omega}_{0}\right)
$$

where $\boldsymbol{\delta}\left(\boldsymbol{\omega}-\boldsymbol{\omega}_{\mathbf{0}}\right)$ is an impulse function, as illustrated in Fig. 5(g)-(i). Similar to the frequency spectrum shift, this operation can be expressed as [58].

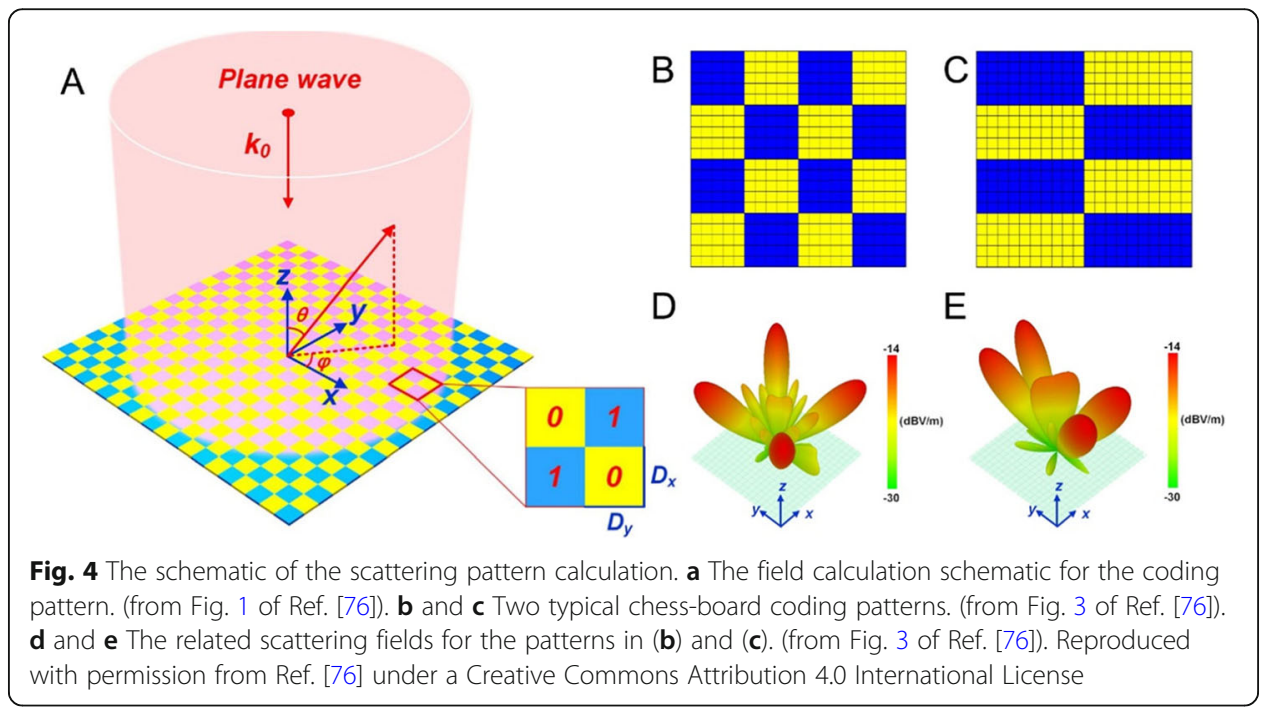




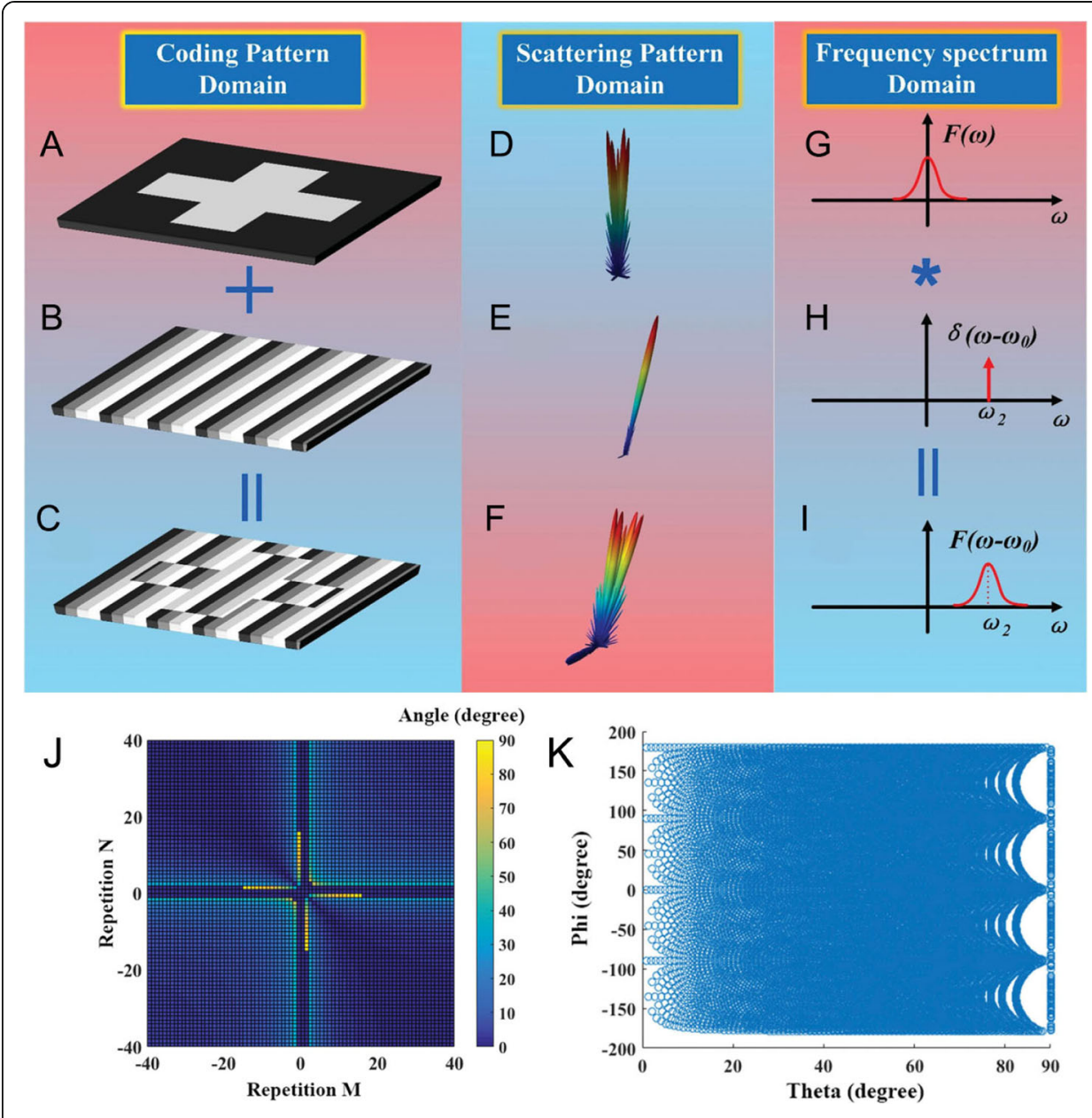

Fig. 5 The convolution theorem for the information metamaterials. a-c The coding patterns operation on a 2-bit metasurface. (from Fig. 1 of Ref. [58]). d-f The related scattering fields. (from Fig. 1 of Ref. [58]). g-i The expression in the frequency spectrum domain. (from Fig. 1 of Ref. [58]). $\mathbf{j}$ The calculated deflection angles for the diverse coding sequence periods. (from Fig. 5 of Ref. [58]). $\mathbf{k}$ The possible scattering directions for 2bit information metamaterials. (from Fig. 5 of Ref. [58]). Reproduced with permission from Ref. [58] under the terms of the Creative Commons Attribution License. Copyright 2016, WILEY-VCH Verlag GmbH \& Co. KGaA, Weinheim

$$
\mathrm{E}\left(x_{\lambda}\right) \cdot e^{j x_{\lambda} \sin \theta_{0}} \stackrel{F F T}{\Leftrightarrow} E(\sin \theta) * \delta\left(\sin \theta-\sin \theta_{0}\right)=E\left(\sin \theta-\sin \theta_{0}\right)
$$

in which $e^{j x_{\lambda} \sin \theta_{0}}$ describes the electric field generated by a gradient phase pattern. Relative to the spectrum shift in the frequency domain, the above equation indicates the spatial shift of the scattering beams, namely the coding convolution operation for information metamaterials. More importantly, this coding sequence operation provides more elaborate tailoring on the scattering angle, which overcome the discontinuity induced by coding discretization. The desired angle calculation can be obtained following the equation below,

$$
\left\{\begin{array}{c}
\theta=\sin ^{-1}\left(\sqrt{\sin ^{2} \theta_{1} \pm \sin ^{2} \theta_{2}}\right) \\
\phi=\tan ^{-1}\left(\sin \theta_{1} / \sin \theta_{2}\right)
\end{array}\right.
$$

where $\theta$ and $\phi$ are the desired scattering angles, $\theta_{1}$ and $\theta_{2}$ are the scattering angles of 
operator sequences. To show its manipulating range, the authors analyzed the manipulating range with a 2-bit coding metasurface, as presented in Fig. 5j and k. In Fig. 5j, the deflection angles of the scattering beams almost cover from $0^{\circ}$ to $90^{\circ}$, when the period ( $\mathrm{M}$ and $\mathrm{N}$ ) of the coding sequence varies from 0 to 40 . Figure $5 \mathrm{k}$ further discusses the possible scattering directions in the upper-hemisphere, indicated by the $\mathrm{x}$ and $y$-axis. We can observe that the attainable angles cover the upper half space.

The entropy in the information metamaterials

To measure the amount of information from the material perspective, Cui et al. have proposed definitions of entropy for coding metasurface [59]. In previous theory, the information system is composed of transmitters, receivers and channels, where the Shannon entropy is used to estimate the information in such system [95]. Similar system is also established on metasurfaces and horn antennas, as exhibited in Fig. 6a and b. When considering a general 1-bit coding metasurface, we only have four selections for adjacent codes $G(0,1), G(0,1), G(0,1)$ and $G(0,1)$, as shown in Fig. 6c. Referring to the normalized Shannon entropy, the author proposed the geometrical entropy and physical entropy to describe the information carried by the coding pattern and far-field pattern respectively, as shown in Fig. 6d. The expressions of the geometrical entropy $\left(\mathrm{H}_{1}\right)$ and physical entropy $\left(\mathrm{H}_{2}\right)$ are defined as [59]

$$
\begin{aligned}
& H_{1}=-N^{-1} \sum_{x} P(x) \log _{2} P(x) \\
& H_{2}=-\sum_{i=1}^{2} \sum_{j=1}^{2} P_{i j} \log _{2} P_{i j}
\end{aligned}
$$

in which $N$ is the number of coding units and $P(x)$ represents the corresponding probability, $P_{i j}$ is the joint probability of a group $\mathrm{G}(\mathrm{i}, \mathrm{j})$ representing two adjacent coding elements. To clearly distinguish the entropy variation, a random diffusion is applied on a 1-bit coding pattern, as shown in Fig. 6e-g. In Fig. 6h, as the diffusion process deepens, the entropy value also increases, in which the geometrical entropy and physical entropy approaches nearly the same in the end. More interestingly, such a transformation is similar to the entropy definition in thermodynamics, suggesting some potential relationships.

\section{Space-time-coding modulation systems based on information metamaterials}

In above illustrations, the digital coding and programmable metamaterials are focused on the spatial arrangement of coding patterns. As another important dimension, the temporal modulation has attracted great attention in metasurface researches [77, 85, 96, 97]. To extend the digital coding to temporal dimension, time-coding and more general space-time-coding digital metamaterials have been presented. As an important property of the information metamaterials, the programmable coding element not only brings the editable functions but also enables modulation in the temporal dimension. By integrating with FPGA, the coding unit can be operated with a periodical-varied signal, namely bias voltage for PIN diodes. In this section, we introduce three representative works of time-varying modulators based on the programmable metasurfaces. 


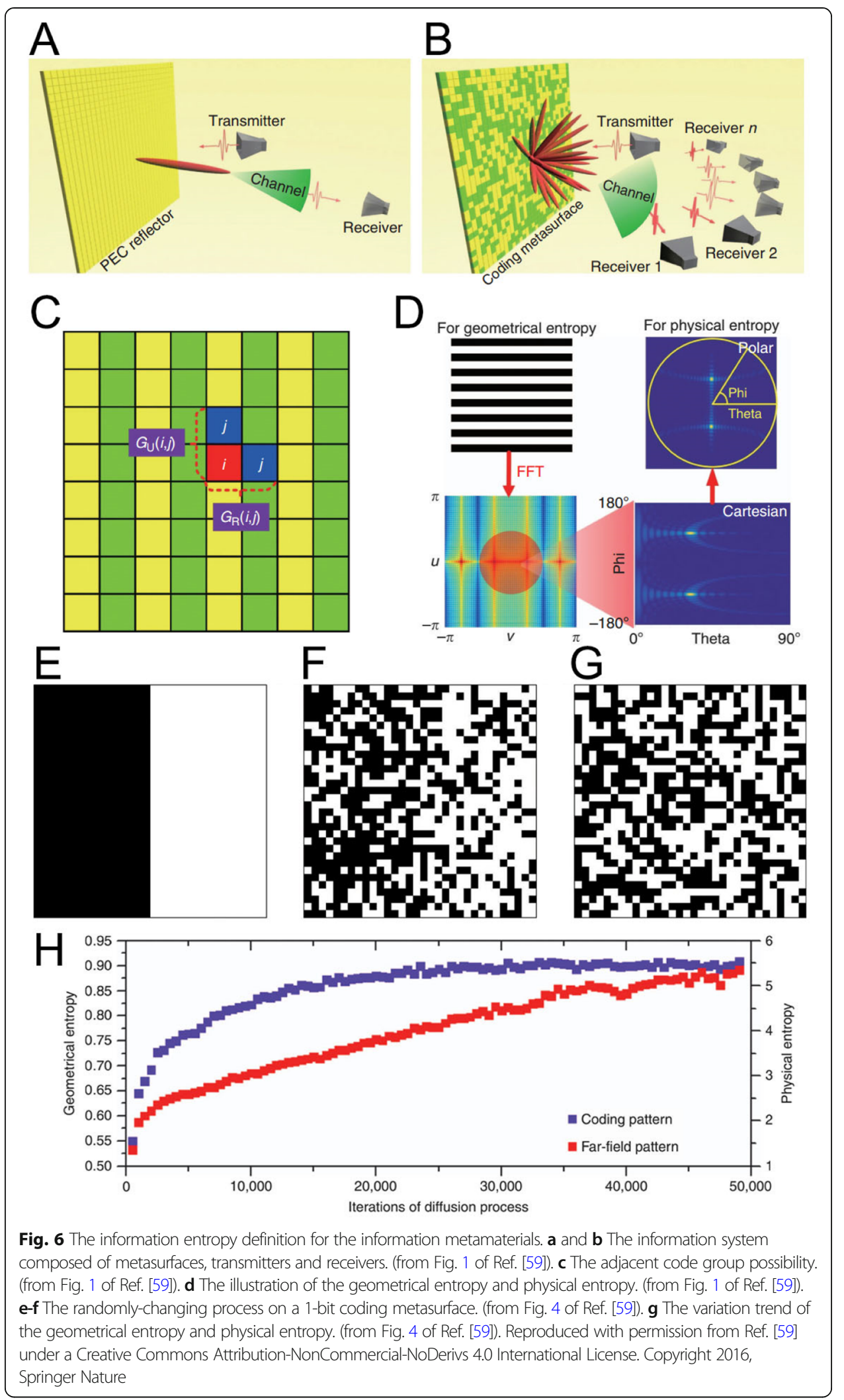




\section{Space-time-coding metasurfaces}

In Ref. [77], Zhang et al. firstly proposed a space-time-coding digital metasurface, by combining a programmable metasurface and FPGA temporal-control, as depicted in Fig. 7a. When illuminated by a plane-wave of frequency $f_{c}$, for a pattern-fixed metasurface, the scattering energy still remains at $f_{c}$. However, when the coding pattern varies in the time domain periodically with a specific frequency $f_{0}$, as illustrated in Fig. $7 \mathrm{a}$, the scattering beams have different responses in harmonic frequencies $f_{c}+m f_{0}$. For different harmonic points, the scattering directions are distinct. The authors presented a scattered field calculation for the space-time coding metasurface at the $m$ th harmonic frequency $f_{c}+m f_{0}$, which is written as [77]

$$
F_{m}(\theta, \phi)=\sum_{q=1}^{N} \sum_{p=1}^{M} E_{p q}(\theta, \phi) \exp \left\{j \frac{2 \pi}{\lambda_{c}}\left[(p-1) d_{x} \sin \theta \cos \phi+(q-1) d_{y} \sin \theta \cos \phi\right]\right\} a_{p q}^{m}
$$

where $d_{x}$ and $d_{y}$ are the element periods along the $\mathrm{x}$ and $\mathrm{y}$ directions, respectively, and $\lambda_{c}$ is the central operational wavelength; $a_{p q}^{m}$ is the Fourier series coefficients of the time-domain periodic function, written as

$$
a_{p q}^{m}=\sum_{n=1}^{L} \frac{\Gamma_{p q}^{n}}{L} \operatorname{sinc}\left(\frac{\pi m}{L}\right) \exp \left[\frac{-j 2 \pi(2 n-1)}{L}\right]
$$

With the above method, the space-time coding pattern is able to generate arbitrary propagation directions. Figure $7 \mathrm{~b}$ and $\mathrm{c}$ exhibit a space-time pattern for the beamdeflection, producing a field listed in Fig. 7d. More interestingly, the 2-bit space-time coding pattern can be equivalent to a 3-bit spatial coding pattern when employing the time-varying modulation, as illustrated in Fig. 7e. With more bits, the scattering patterns can be designed in higher precision. Except for the manipulation on beamdeflection, the space-time coding metasurface is also capable of beam shaping, and scattering-signature control. In Fig. 7f, an orbital angular momentum forming pattern is presented. And Fig. $7 \mathrm{~g}$ shows a calculated pattern of the OAM beam.

In addition to the beam-steering, time-varying approaches are applied for breaking the reciprocity [85, 97]. As presented in Fig. 8a, a non-reciprocal transmission is realized on 2-bit programmable metasurface, validating the method through space-time coding. For a reciprocal reflective metasurface, the relative angle does not change whether it is in the forward or reverse incidence. In contrast, once the pattern is implemented with a time-varying modulation, the forward incidence of $f_{1}$ at the angle $\theta_{1}$ is reflected to at $f_{2}$. According to the symmetry, when a reverse incidence of $f_{2}$ comes at the angle $\theta_{2}$, the reflected wave does not return to $f_{1}$ at the angle $\theta_{1}$, but to a new angle $\theta_{3}$ at a new frequency $f_{3}$, which obviously breaks the time-reversed symmetry. Such kind of system is classified as a non-reciprocal system. Figure $8 \mathrm{~b}$ and c exhibit the fabricated metasurface and experimental environments, where the metasurface is excited and received with linear-polarized horn antenna, and the phase pattern is fully controlled by FPGA. The measured results are listed in Fig. 8d and e, from which the nonreciprocal transmission is clearly observed. 


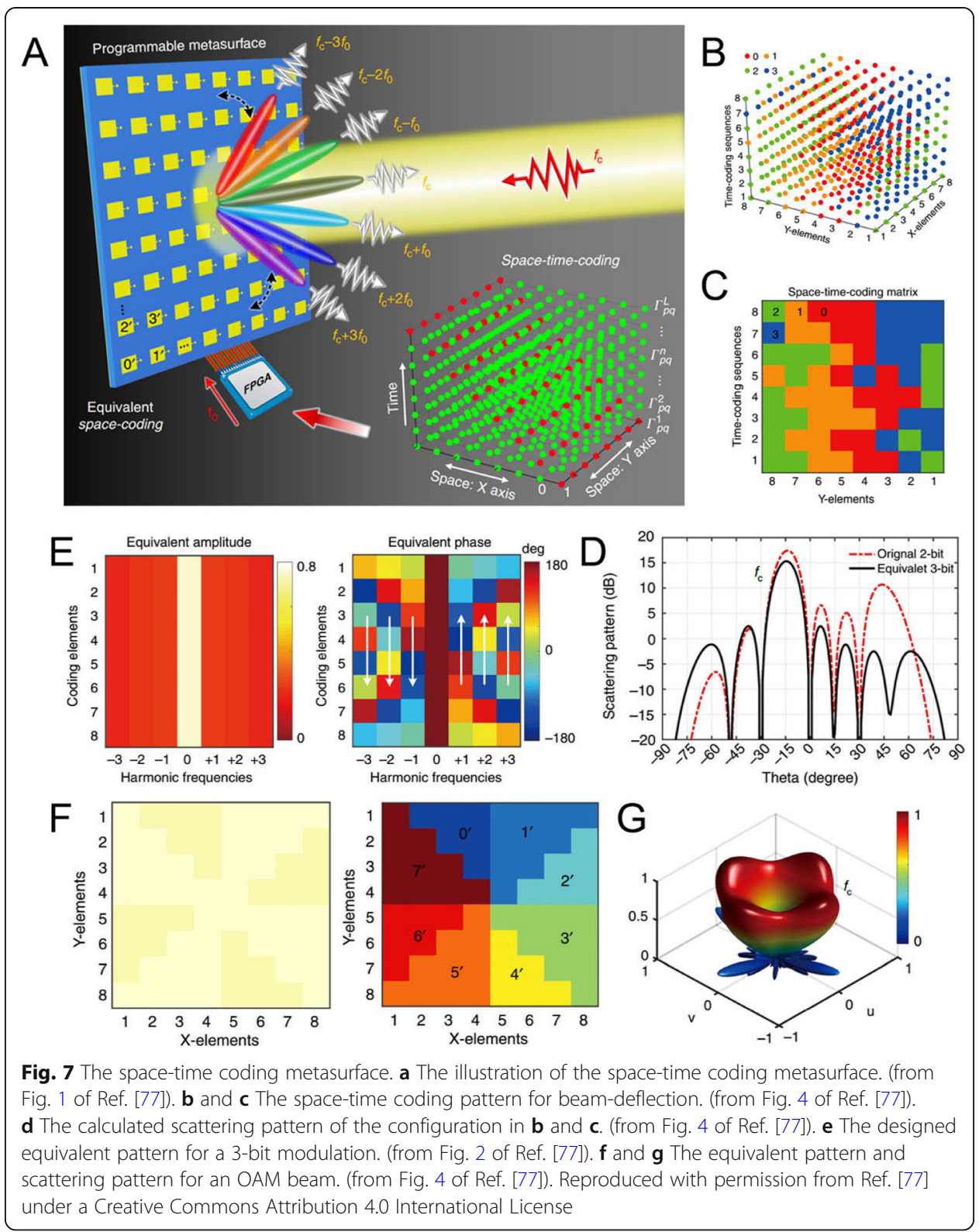

\section{Time-domain manipulator on harmonics}

The independent phase-amplitude control is a challenge for ultrathin metasurface. The integration of active devices in such a thin structure can easily cause mutual interaction between reflected amplitude and phase, as well as extra losses. But for time-modulating digital coding metasurfaces, the harmonic offers another important method to tailor on amplitude and phase responses. In Ref. [78], Dai et al. presented an independent control on both parameters with space-time coding metasurfaces, as presented in Fig. 9a. To realize the programmable property, a reflected element embedded with four varactors (SMV-2019, Skyworks) is designed in Fig. 9b, providing a phase range of more than $270^{\circ}$. The bias voltage is implemented through the transverse metal belt, which is 

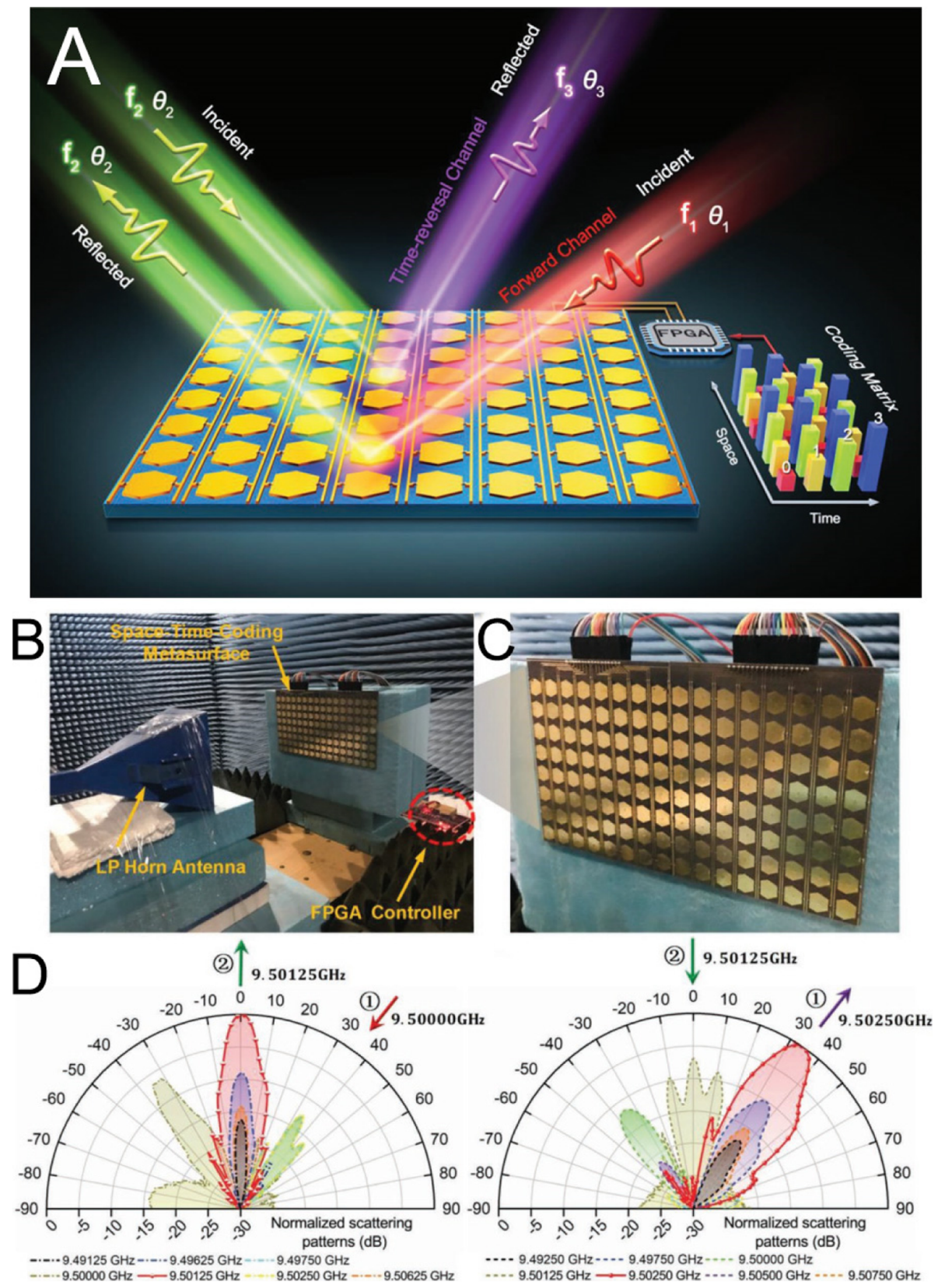

Fig. 8 The non-reciprocal transmission achieved by space-time coding metasurface. a The schematic of the non-reciprocal transmission obtained by a time-varying coding pattern. (from Fig. 1 of Ref. [85]). $\mathbf{b}$ and $\mathbf{c}$ The fabricated metasurface sample and the measurement environment. (from Fig. 6 of Ref. [85]). $\mathbf{d}$ and $\mathbf{e}$ The measured scattering fields at different harmonic frequencies. (from Fig. 6 of Ref. [85]). Reproduced with permission from Ref. [85]. Copyright 2019, WILEY-VCH Verlag GmbH \& Co. KGaA, Weinheim

connected with the top patch via holes. As the varactor capacitance alters, the phase and magnitude are dependent on each other. To achieve independent control, a new degree of freedom needs to be induced. Before the final deviation, the authors firstly selected two phase responses $\phi_{1}$ and $\phi_{2}$. Then the spectral expression for the reflected wave is written as 

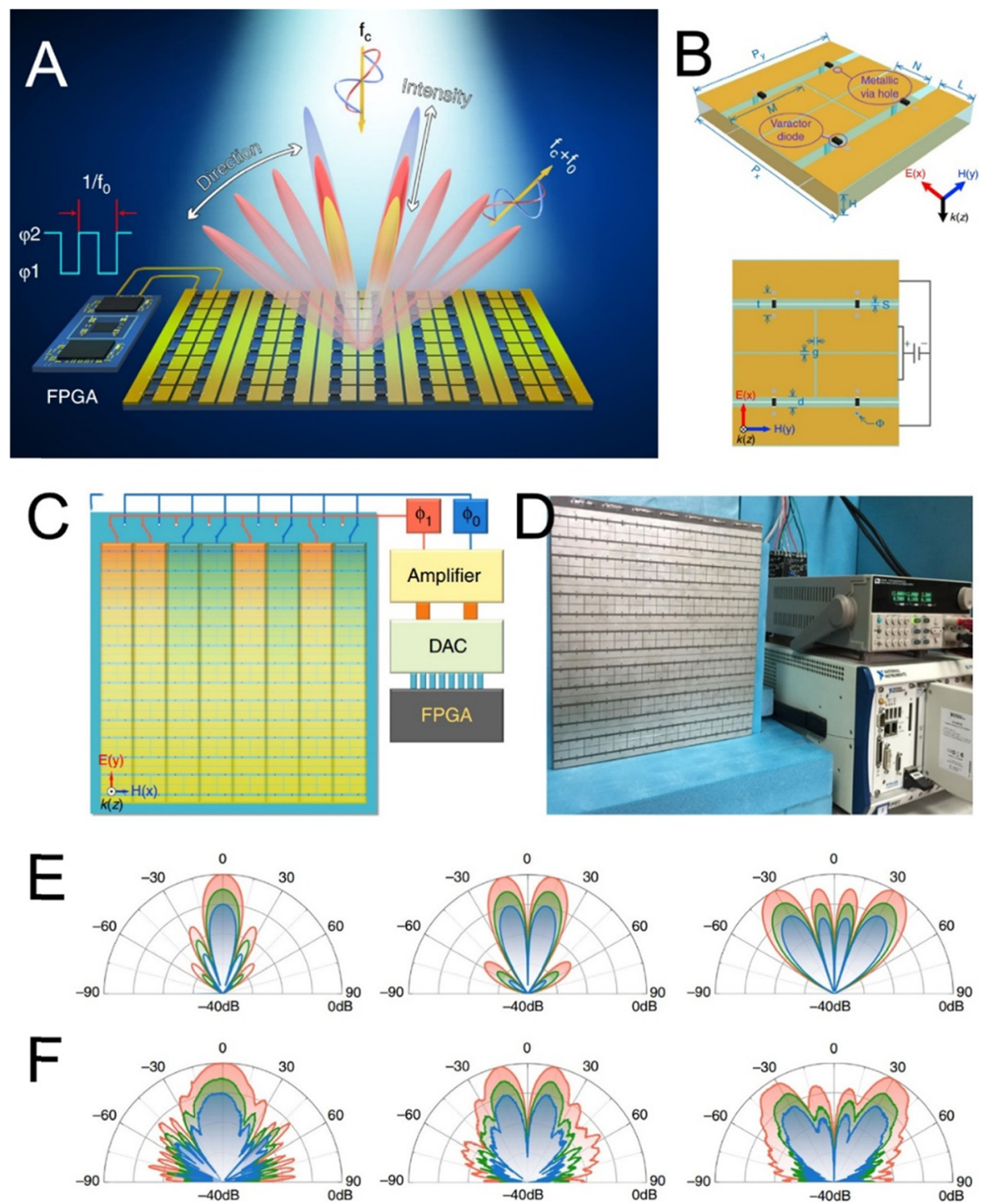

Fig. 9 The time-domain modulator on harmonics. a The schematic of the time-domain information metamaterials. (from Fig. 1 of Ref. [78]). $\mathbf{b}$ The designed programmable element. (from Fig. 2 of Ref. [78]). c The configuration of the metasurface. (from Fig. 3 of Ref. [78]). $\mathbf{d}$ The fabricated sample and the measurements. (from Fig. 3 of Ref. [78]). $\mathbf{e}$ and $\mathbf{f}$ The simulated and measured results for the independent control on phase and amplitude. (from Fig. 5 of Ref. [78]). Reproduced with permission from Ref. [78] under a Creative Commons Attribution 4.0 International License. Copyright 2018, Springer Nature

$$
\begin{aligned}
E_{r}(\omega)= & 2 \pi A \cos \frac{\phi_{2}-\phi_{1}}{2} e^{j \frac{\phi_{2}+\phi_{1}}{2}} E_{i}(\omega) \\
& +\sum_{m=-\infty}^{+\infty} \frac{4 A}{2 m-1} \sin \frac{\phi_{2}-\phi_{1}}{2} e^{j\left[\frac{\phi_{2}+\phi_{1}}{2}-(2 m-1) \omega_{0} t_{0}\right]} E_{i}\left[\omega-(2 m-1) \omega_{0}\right]
\end{aligned}
$$

Furthermore, the amplitude and phase of the $k^{\text {th }}$-order harmonic are summarized as 


$$
\begin{aligned}
E_{r}\left(k \omega_{0}+\omega_{c}\right) & =|A| \angle \Phi \\
& =\left\{\begin{array}{c}
\left|A \cos \frac{\phi_{2}-\phi_{1}}{2}\right| \mathrm{e}^{\left.\mathrm{j} j \frac{\phi_{2}+\phi_{1}}{2}+\pi\left[\varepsilon\left(\frac{\phi_{2}-\phi_{1}-\pi}{2}\right)+\varepsilon\left(\frac{\phi_{1}-\phi_{2}-\pi}{2}\right)\right]\right\}} \mathrm{k}=0 \\
\left|\frac{2 A}{k \pi} \sin \frac{\phi_{2}-\phi_{1}}{2}\right| \mathrm{e}^{\mathrm{j}\left\{\frac{\phi_{2}+\phi_{1}}{2}-\pi\left[\varepsilon\left(\phi_{2}-\phi_{1}\right)+\varepsilon(\mathrm{k})\right]-\mathrm{k} \omega_{0} t_{0}\right\}} \mathrm{k}= \pm 1, \pm 3, \pm 5 \ldots \\
0 \mathrm{k}= \pm 2, \pm 3, \pm 4 \ldots
\end{array}\right.
\end{aligned}
$$

Within the above calculation, the extra phase shift is obtained as another dimension, which is totally independent of the element features. Hence, the synthetic manipulation has independent manipulation on both phase and amplitude. Figure $9 \mathrm{c}$ gives the configuration of the designed metasurface, where a digital-analog conversion (DAC) module and an analog amplifier module are employed for varactor control. The fabricated metasurface and the measurement arrangement are shown in Fig. 9d. The simulated and measured results are listed in Fig. 9e and f, respectively, showing good agreements on the modulation performance.

\section{Wireless communication systems based on information metamaterials}

One fascinating application of the information metamaterials is communication, which has been actualized on the programmable metasurfaces with two distinct architectures. In the traditional communication systems, the digital data is successively processed by DAC, mixer, amplifier, filter, and finally transmitted by antenna. In the receiving system, a similar process is executed again to obtain the original data. Such a communication system requires the integration of multiple components and high systemcomplexity. To reform the traditional transceiving mechanism, Cui et al. proposed a direct transmission architecture based on the programmable coding metasurface [79], which significantly simplifies the system complexity. As shown in Fig. 10a, the proofof-principle system is composed of the programmable metasurface, FPGA and feed source. By arranging the specific codes from FPGA, the metasurface reflects the energy to the specific directions, where several receivers are located at several fixed locations. The energy detected by the specific receiver represents the distinct information data, which directly establishes the communication mechanism. On the basis of this system, the authors designed a series of coding sequences such as "01010101", to produce the scattering pattern, encoding as the transmitting codes. The manufactured prototype is shown in Fig. 10b, as well as a customized FPGA is given in Fig. 10c.

The main challenge of this communication system is channel noise restraining, since some scattering patterns are difficult to distinguish. A self-optimization is firstly performed to select appropriate radiation patterns, namely transmitting codes. To maintain the system bit error ratio under an acceptable range, the authors applied the order maximum modulation of the quadrature phase shift keying modulation [98]. In Fig. 10d and e, the measured results are given, in which a binary image of Lena is transferred from transmitter to receiving horns. It is clearly observed that the image is almost perfectly recovered, verifying its performance. Moreover, communication systems with such distributed receivers are also practicable for secret communications.

Except for the spatial energy redistribution, another communication framework is relied on the time-domain coding metasurface, proposed by Zhao et al. [80]. As we introduced above, the temporal modulation promises desirable control on both phase and 


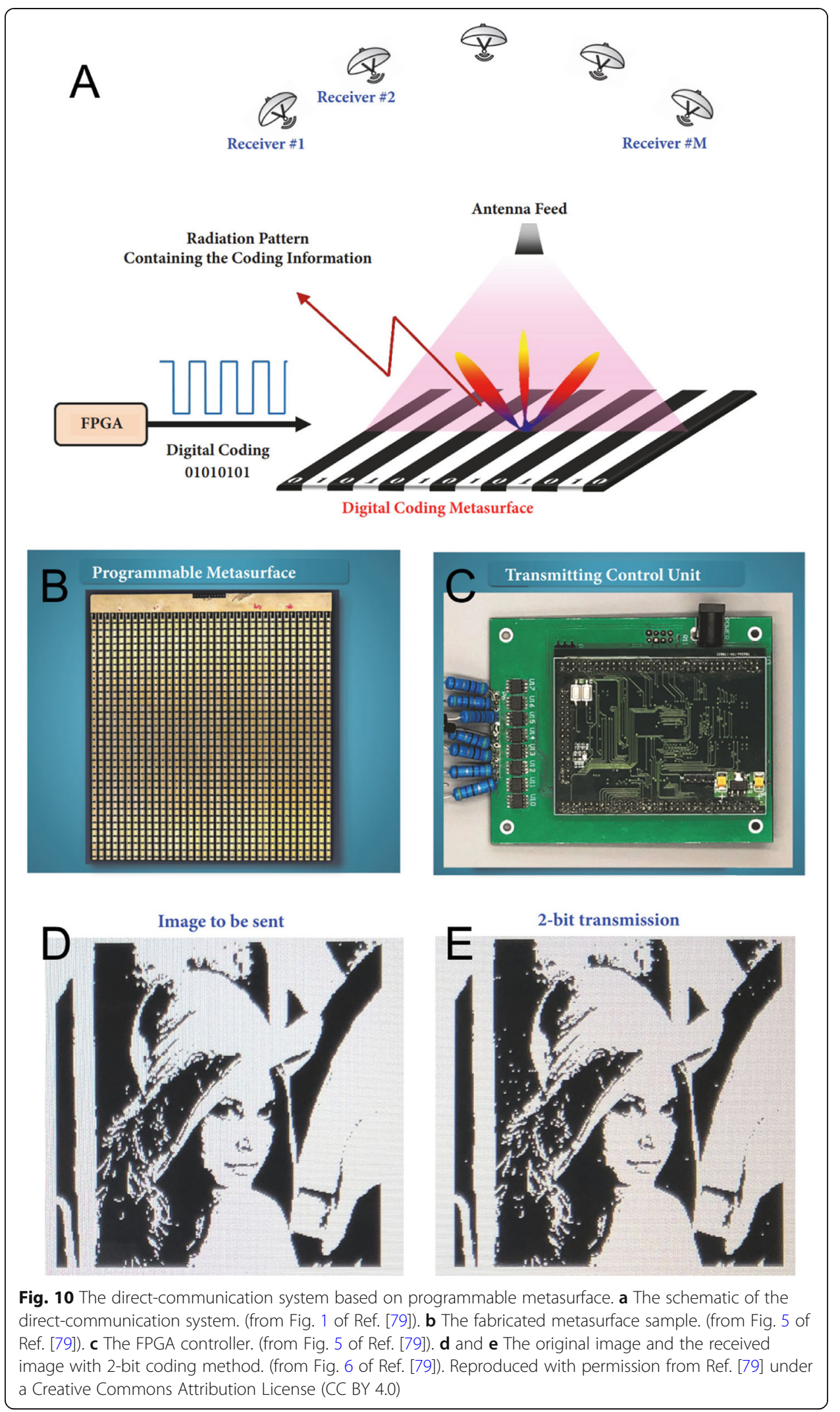


amplitude of harmonics, which is naturally applied for information representing. Benefited from the programmable metasurface, such a communication mechanism similarly demands low system complexity and cost. As presented in Fig. 11a, a reflective metasurface embedded with varactor diodes (SMV2019) is designed. The elements in each column are driven with the same voltage, through via holes and transverse belts. When illuminated by an incidence of $f_{c}$, the metasurface with time-varying pattern reflects a field of $E_{r}(f)$, which is written as

$$
E_{r}(f)=a_{0} E_{i}(f)+\sum_{k=1}^{\infty}\left[a_{k} E_{i}\left(f-k f_{0}\right)+a_{-k} E_{i}\left(f+k f_{0}\right)\right]
$$

where $f_{0}$ is the modulation frequency of varactor diode, as well as the coding pattern. In the traditional communication system, these $\pm k^{t h}$-order harmonics are the serious

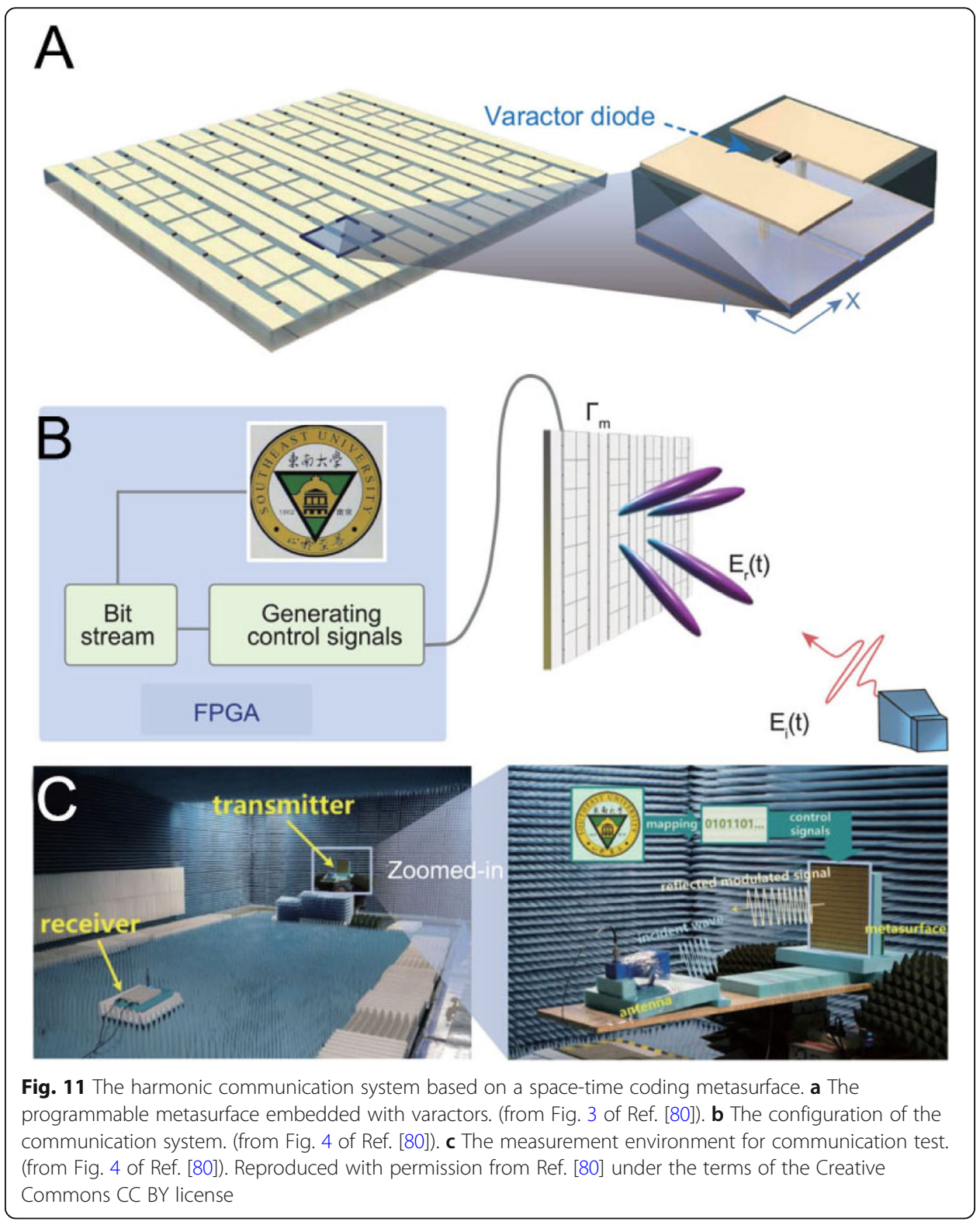


interference for channels, which need to be suppressed as much as possible. On the contrary, the authors used these "interference" to transmit the coding information and explore implementation of a binary frequency-shift keying (BFSK) coding method, by synthesizing two basic carrier frequencies. Four 2-bit coding sequences are designed as “02020202", “01010101”, “01230123”, “32,103,210”, having distinct amplitude responses on the and order harmonics. The configuration of the binary phase shift keying transmitter is exhibited in Fig. 11b, only composed of a programmable metasurface and FPGA for the data process. Comparing to the traditional heterodyne architecture, the metasurface and FPGA in the present system replace the complicated DAC, analogdigital conversion and a series of RF components, suggesting better simplicity and efficiency. In measurements, as shown in Fig. 11c, the transmitter fixed on a rotatable table emits a picture, while a receiver composed of a soft-defined radio receiver (NI USRP RIO 2943R) and an omnidirectional antenna demodulates the information.

\section{Programmable imaging systems}

Relative to the wireless communications, imaging is another popular application, which has been realized from microwave to visible regime [99-102]. Among the plentiful methods, metasurfaces yield more advantages because of its unprecedented capability on phase and amplitude control of propagation wave. Besides, the subwavelength dimension offers a higher resolution and efficiency than the traditional holograms, which are restricted by the disturbing Floquet modes. Moreover, the simplified structure and ultrathin profile bring the metasurfaces with more application potentials. However, most previous metasurfaces were designed with static images or functions, which manifestly hinders its development. To solve that defect, imagers and holograms based on the programmable metasurfaces have been proposed with customizable features [8183]. In this section, we discuss three imagers made of information metamaterials, to realize the single-sensor single-frequency imaging, reprogrammable holograms, and machine-learning imaging, respectively.

\section{Single-sensor single-frequency imager}

The conventional imaging system usually requires a sensor array to detect discrepant scattering information, to sequentially resolve the matrix. To simplify the system framework, the concept of single-sensor imaging is proposed in 2008 [103]. But this kind of imager requires reconfigurable patterns or radiation modes for solving the coefficient equation. This requirement can be exactly satisfied with programmable metasurfaces. $\mathrm{Li}$ et al. presented a single-sensor single-frequency imager using a transmissive programmable metasurface [83], as shown in Fig. 12a. Two PIN diode is integrated on each unit cell, leading to four different response states as " 00 ", " 01 ", " 10 ", and " 11 ". The relationship between objects and measured data can be described with the following coefficient matrix

$$
\left[\begin{array}{c}
V^{(1)} \\
V^{(2)} \\
\vdots \\
V^{(P)}
\end{array}\right]=\left[\begin{array}{cccc}
G_{11} & G_{12} & \ldots & G_{1 N} \\
G_{21} & G_{22} & & \\
\vdots & & \ddots & \\
G_{P 1} & & & G_{P N}
\end{array}\right]\left[\begin{array}{c}
\sigma_{1} \\
\sigma_{2} \\
\vdots \\
\sigma_{N}
\end{array}\right]
$$

where $\sigma, G$, and $V$ are object-area vector, system response matrix and measurement 

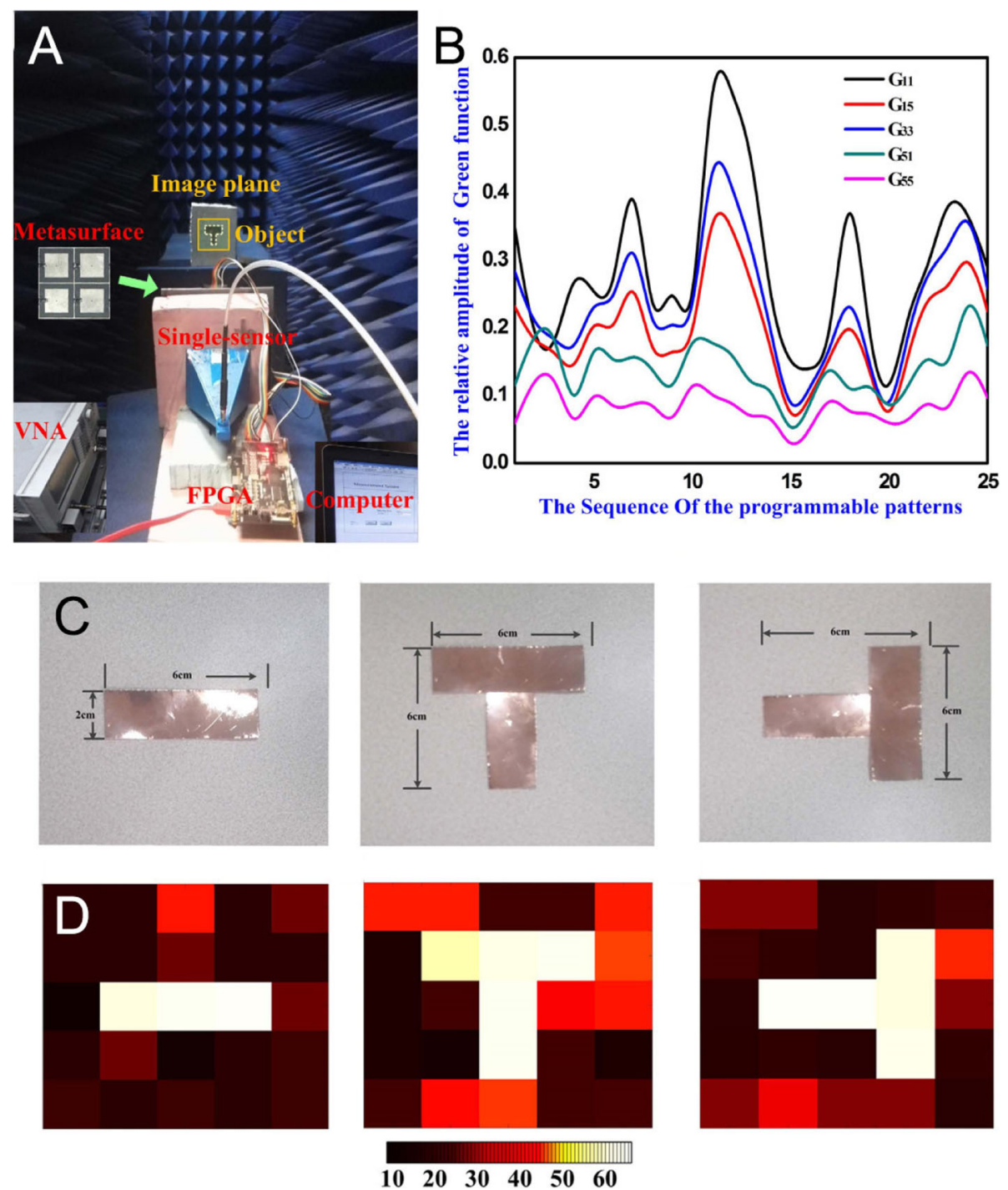

Fig. 12 The single-sensor single-frequency imager based on a 2-bit programmable metasurface. a The fabricated metasurface and the measurement configuration. (from Fig. 4 of Ref. [83]). b The sampled information of the generalized system response matrix. (from Fig. 4 of Ref. [83]). c Three metallic objects for imaging. (from Fig. 4 of Ref. [83]). d The measured imaging results for the objects in (C). (from Fig. 4 of Ref. [83]). Reproduced with permission from Ref. [83] under a Creative Commons Attribution 4.0 International License

results respectively. Since the metasurface is composed of $25 * 25$-unit array, $P$ and $N$ are set as 25 , and the matrix is composed of $25 * 25=625$ units. Logically, the above matrix operation is expressed as $\boldsymbol{V}=\boldsymbol{G} \boldsymbol{\sigma}$, and $\boldsymbol{\sigma}=\boldsymbol{G}^{-\mathbf{1}} \boldsymbol{V}$, which means the sub-object area number determines the measurement time. Fortunately, a diverse radiation pattern is switched easily with the programmable metasurface and FPGA. By applying the scattering field calculation, the final results of imaging are easily obtained.

Figure 12a illustrates the measurement environment of the presented imager, which is illuminated by a single horn antenna. The coding pattern on the metasurface is controlled by FPGA and computer. By varying the on-off patterns of diodes, the relative amplitude of Green function is changed distinctively, as shown in Fig. 12b. Three 
imaging objects, made from thin copper layers, are presented in Fig. 12c. And the final measured results are showed in Fig. 12d, suggesting good consistency with objects.

\section{Reprogrammable metasurface hologram}

Hologram is one of the fascinating technologies to encode the image information on the specific media. Some metasurface holograms have been proposed with full-color [104], high efficiency and resolution [105]. Although metasurfaces yield many advantages on the holograms, most works on metasurfaces are passive and immutable, impeding its function extension. Before the presented work, alterable hologram still encounters the challenges of active device integration and regulation circuit design. To promote this situation, $\mathrm{Li}$ et al. presented a programmable hologram based on the information metasurface [81]. As illustrated in Fig. 13a, a coding element $\left(6 * 6 * 2 \mathrm{~mm}^{3} \sim\right.$ $0.156^{*} 0.156^{*} 0.05 \lambda^{3}$ ) inserted with a PIN diode is applied [56] to build up a phasemodulating pattern. Similar to the phase-programmable metasurfaces we discussed above, when the DC bias voltage controlled by FPGA changes, the phase pattern on the metasurface is altered as well. Profit from such architecture, the hologram design is simplified into the optimization on the coding patterns. Inspired by the continuous Gerchberg-Saxton (GS) algorithm, the authors proposed a modified GS algorithm for a digital coding pattern. All these patterns calculated on the computer are able to be switched in real-time with digital control, as we showed in Fig. 13a.

In experiment demonstration, a metasurface with 20*20 supercells (each supercell contains $5 * 5$ elements, the total dimension is $600 * 600 \mathrm{~mm}^{2}$ ) is fabricated and measured. A broadband horn antenna (from 6 to $14 \mathrm{GHz}$ ) is employed as a feed source, while a standard waveguide probe is used for scanning the image. To fully validate its performance, the authors design 16 letters as "LOVE PKU! SEU! NUS!", related to 16 distinct coding patterns. The measurement results are listed in Fig. 13b, where the text is clearly observed, further validating its feasibility.

\section{Machine-learning imager}

For microwave imagers, switching speed and quality have become a dilemma since the time and resources cannot be duly handled simultaneously $[99,106]$. To address that problem, compressive sensing algorithm is proposed, but demands for greater source consumptions [107]. Recently, such an obstacle has been efficiently tackled by machine-learning techniques $[108,109]$. However, before its direct implementation on the metasurface imager, a rigorous physical configuration needs to be resolved. This situation is mainly resulted from the lack of a fast-switchable, low-cost and highperformance EM-wave manipulation device. Starting from this idea, Li et al. proposed a programmable metasurface imager equipped with a machine-learning algorithm [82]. As illustrated in Fig. 14a, the presented digital metasurface system is trained with various scene samples for producing high-quality images and realizing high-accuracy classification. In the machine-learning theory, the measurement modes are iteratively learned from the training samples so as to collect information characteristics as much as possible $(M \ll N)$, as shown in Fig. 14b. With the aim of dynamic modulations, a reprogrammable element with 2-bit phase states is presented in Fig. 14c. Three PIN diodes are embedded on a metal bar to alter the electric length for distinct phase 


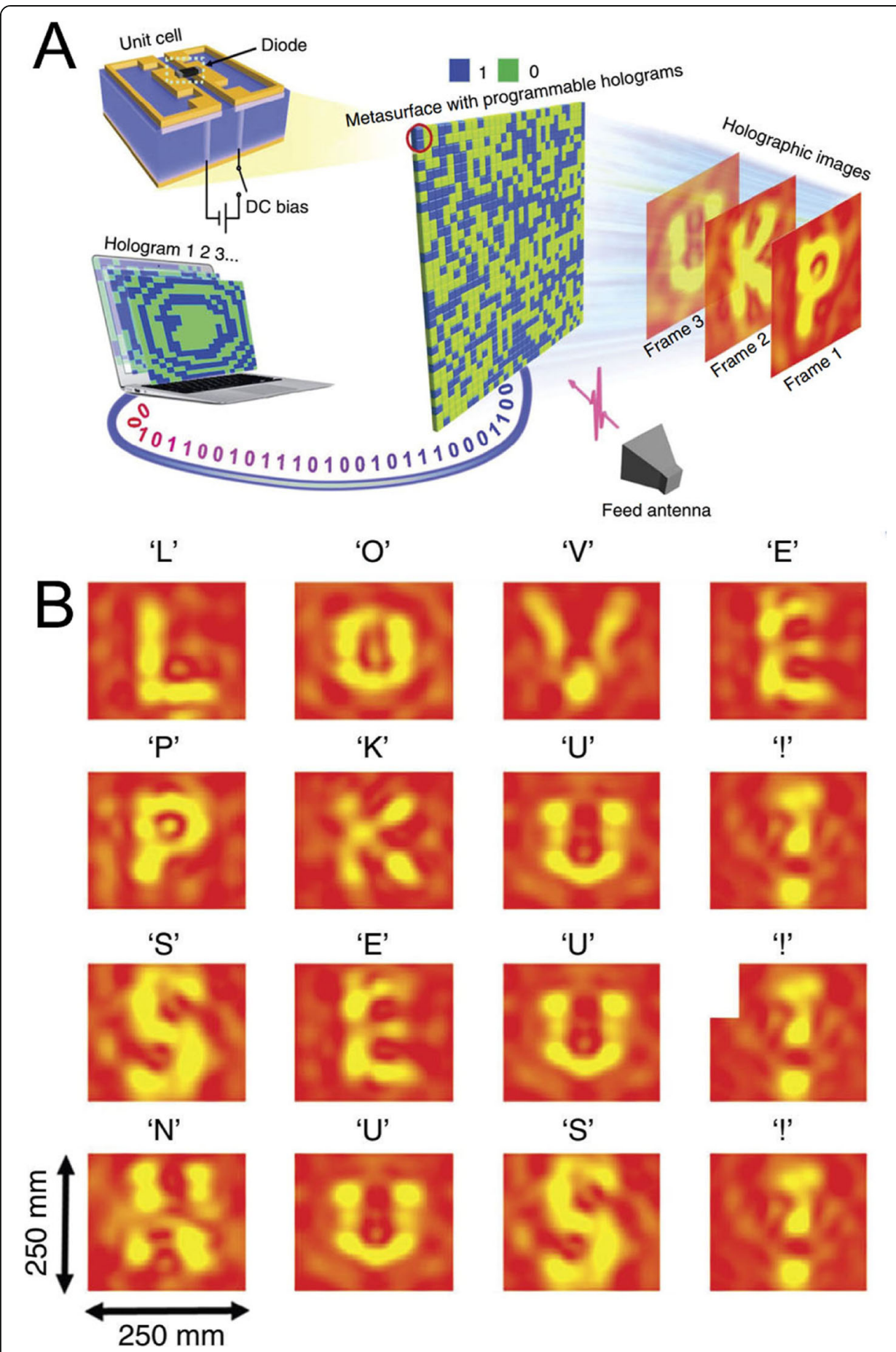

Fig. 13 The programmable holograms based on the information metasurfaces. a The diagram of the programmable holograms using metasurface. (from Fig. 1 of Ref. [81]). b The collected data of the design text images. (from Fig. 4 of Ref. [81]). Reproduced with permission from Ref. [81] under a Creative Commons Attribution 4.0 International License

responses, encoded as " 00 ", " 01 ", " 10 ", and " 11 " states, corresponding to phases of 0 , $90^{\circ}, 180^{\circ}$, and $270^{\circ}$, respectively. Figure $14 \mathrm{~d}$ introduces a demonstration for the realtime programmable metasurface imager, where a moving person behind a wall is displayed. 
In the measurement modes training, a two-step strategy is applied as follows: (1) the radiation patterns are firstly trained with the machine-learning algorithm; (2) the coding patterns are then optimized for the target radiation patterns. For the coding pattern optimization, GS algorithm is applied here to solve the discrete coding problem. Two linear embedding techniques, the random projection [110] and principal component analysis (PCA) [111] are applied here. In Fig. 14e, the experiment environment is shown, in which a testing person is arranged in a microwave anechoic chamber with different poses. After 400 measurements, the imaging results using the machinelearning with PCA and random projection are listed in Fig. 14f and g. We observe that the machine-learning with PCA has better performance and classification correctness than with the random projection. The successful combination between the information

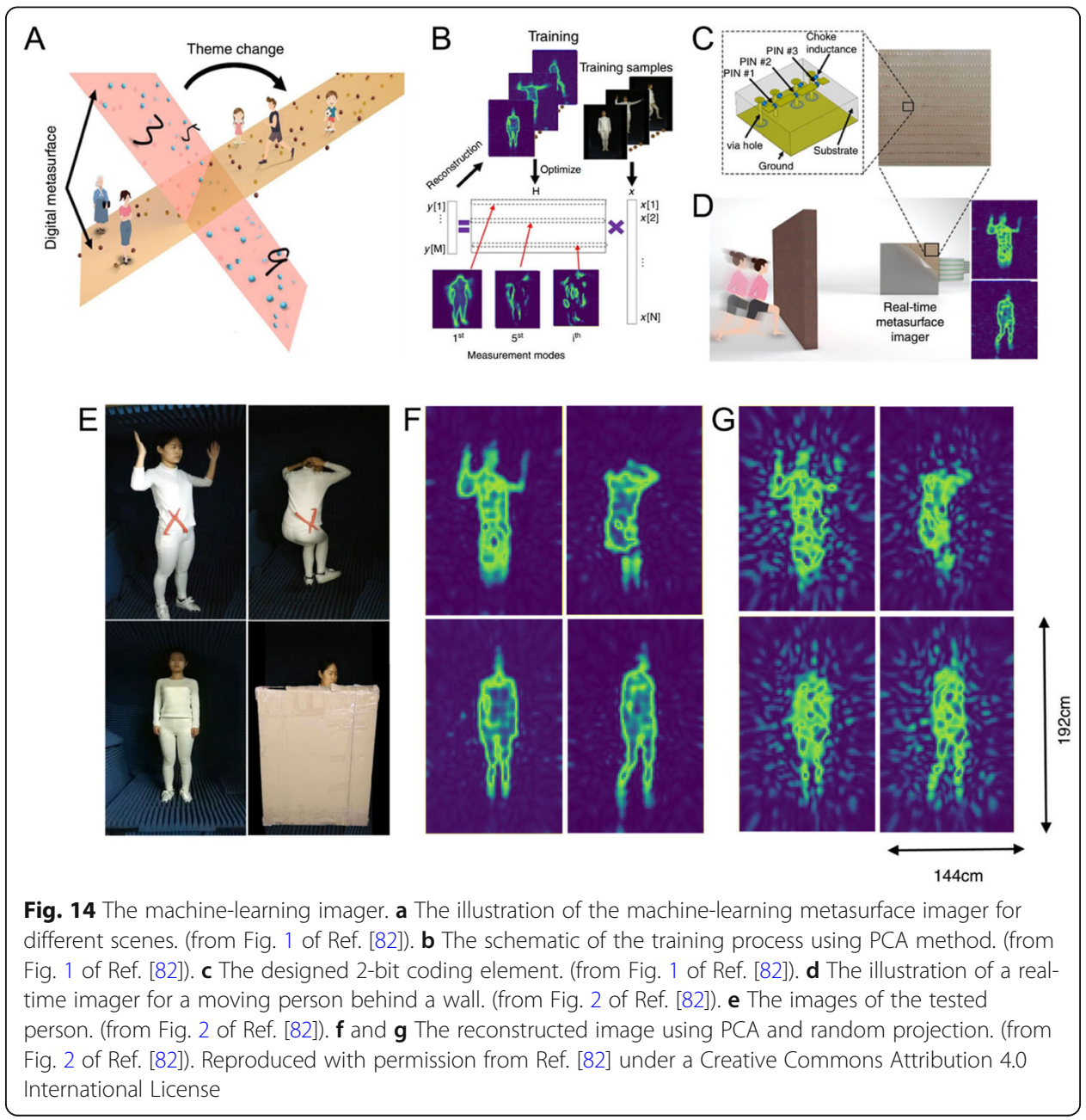


metamaterials and artificial intelligence not only improves the performance, but also offers a new direction for smart devices.

\section{Smart systems based on the information metamaterials}

Looking back on decades of metamaterial developments, though showing multifarious special natures, both traditional metamaterials and digital information metamaterials require human instructions and design. Such architecture is hard to break through the shackles of functions, to achieve intelligent modulation. For metamaterials, especially information metamaterials, intelligence has become an inevitable issue. In our previous articles $[112,113]$, we envisioned that software-defined metamaterials have been fully established on the basis of the programmable units with various functions, while the metamaterials are equipped with learning abilities on the present framework. However, before the learning process, the sensing components need to be integrated with metastructure to collect enough datasets. Hence, in this section, we introduce two original intelligent devices on information metamaterials with unprecedented processing architecture, in which a definition of smart metamaterials will be discussed in detail.

\section{Self-adaptive smart metasurface}

As we discussed above, the previous metamaterial functionalities are all based on the human instructions and pre-designs. Without the pre-defined configuration, the metamaterials are unable to make a decision by itself. Before the self-decision making, the metamaterials should firstly be capable of sensing the surrounding variations. For better understanding, we assume that there is an airborne metamaterial communicating with a satellite, as shown in Fig. 15a. When the attitude and flying direction of airplane change, the metamaterial can self-adaptively adjust its radiation beam to aim to the satellite. To accomplish such intelligence, the metamaterial demands a sensing and feedback channel for decision-making. From this idea, Ma et al. proposed a smart metasurface equipped with a gyroscope sensor to monitor the moving states of the metasurface. The gyroscope returns the angle data on a 3-axis coordinate, and FPGA automatically calculates a coding pattern for desirable focusing directions. In this architecture, two important components, sensor and its micro-controlling unit, are added to complete a processing loop, as illustrated in Fig. 15b. Hence, comparing to the previous programmable metamaterials, smart metasurface no longer requires human instructions with the presented architecture. Most importantly, the authors offered a definition for smart metamaterials as follows. Smart metamaterials are able to initiatively sense the surrounding information, and react to the specific functions according to the predesigned algorithm. To establish such mechanism, a sensor for feedback is necessary to fully close the sensing-feedback-reaction loop for the smart metamaterials.

To realize the aforementioned functions, a 2-bit phase-programmable metasurface is designed and fabricated, as exhibited in Fig. 15b. Two PIN diodes (SMP1320 from SKYWORKS) are integrated on each meta-atom to obtain four distinct phase responses, encoded as " 00 ", " 01 ", " 10 ", and " 11 ". Moreover, a fast feedback algorithm is developed to acquire the coding patterns for arbitrary beam-deflection fields, according to the data from the sensors. FPGA further executes the bias configuration on PIN diode array. Diverse beam functionalities are designed and demonstrated, including single- or 


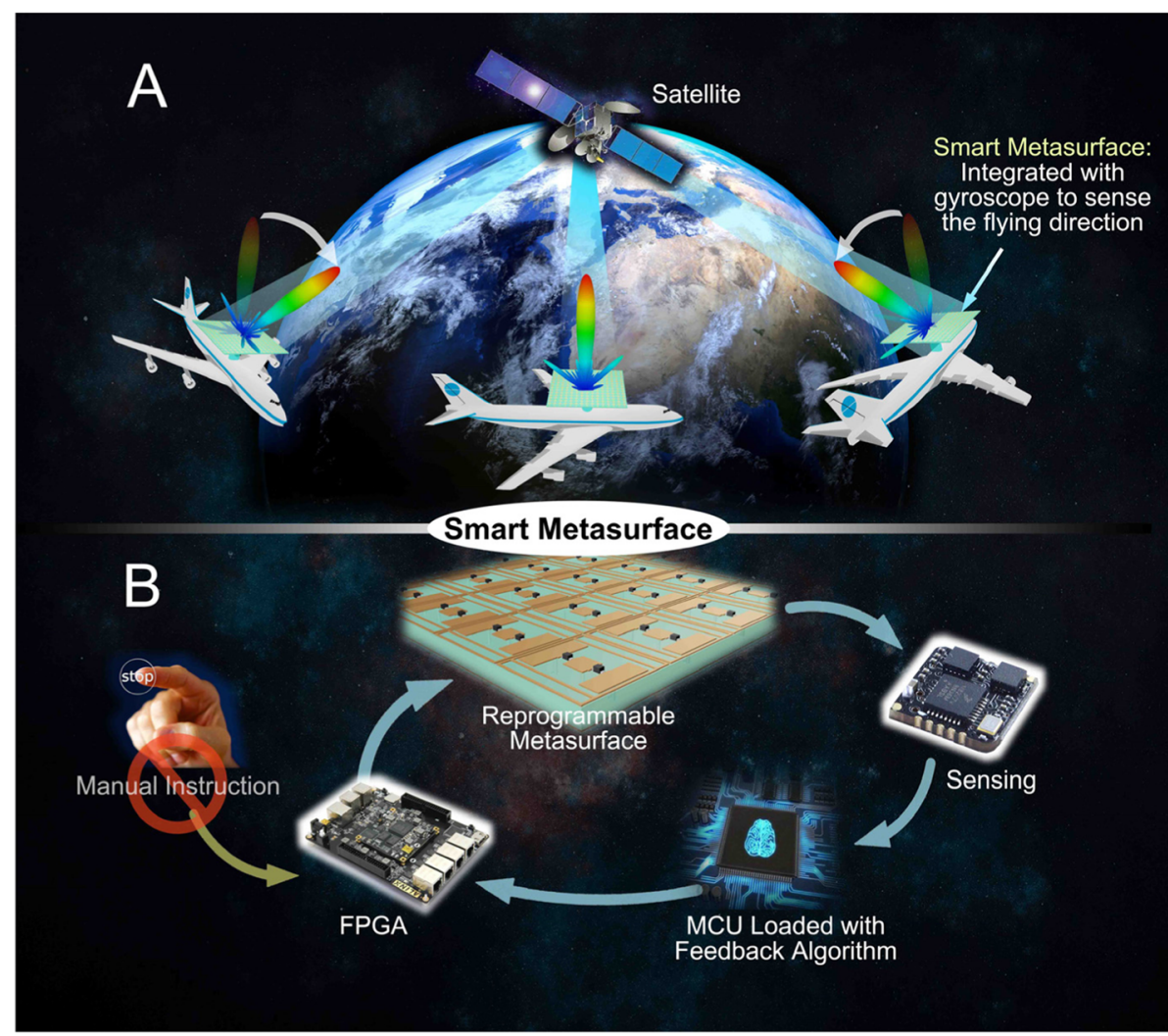

Fig. 15 The schematic of the smart metasurface. a The example situation for self-adaptive smart metasurface. (from Fig. 1 of Ref. [8]). b The illustration of a closed-loop system for smart metasurface. (from Fig. 1 of Ref. [86]). Reproduced with permission from Ref. [86] under a Creative Commons Attribution 4.0 International License. Copyright 2019, Springer Nature

multi-beam deflection, beam scanning, RCS reduction and votex beam. In addition, the presented smart metasurface is promised to extend other sensors on this platform, as depicted in Fig. 16a, where the metasurface is supplemented with the sensors for light, humidity, height and heat. Therefore, more dimensions on the sensing functions can be developed as well. Take the light sensor for example (showed in Fig. 16b and c), when the environment luminance alters, the metasurface switches the scattering pattern from dual-beam to low-scattering, on the basis of the pre-defined algorithm. Figure 16d provides the photograph of the complete samples with multiple sensors, as well as their status. The measured and simulated results in Fig. 16e and f have good consistency with each other, verifying the performance.

\section{Intelligent imager and recognizer}

The development of the Internet of Things has promoted a series of intelligent devices, especially for the in-door space, where the RF signal is a great medium for locating [114], posture sensing and recognition [115-117]. However, these devices are specifically designed into some fixed configuration, which can hardly be redefined for successive tasks. Besides, complex system setup and high-cost hardware further impede its promotion and application. To tackle these challenges, Li et al. presented an artificial 


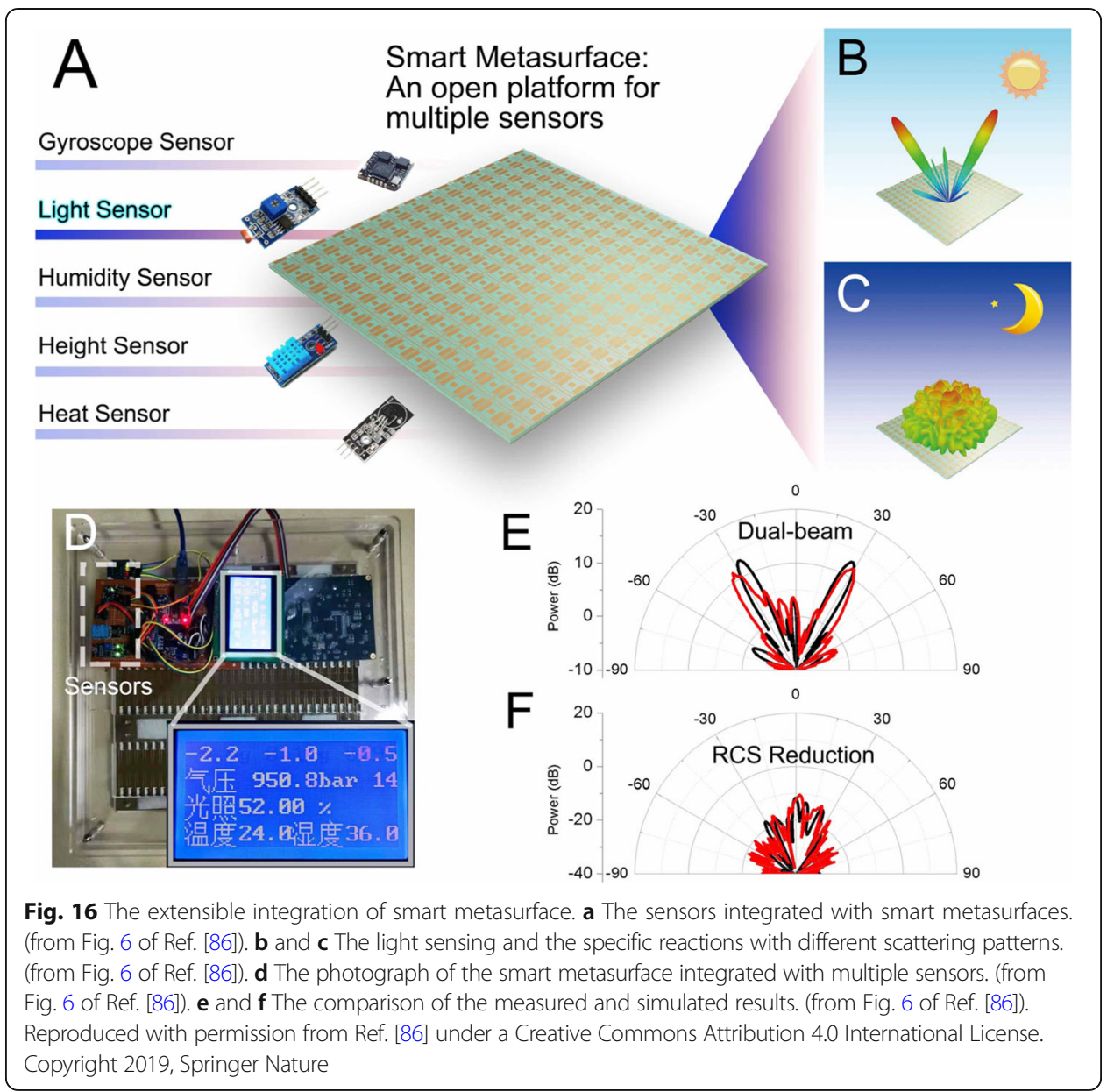

neural network-driven intelligent metasurface for adaptive EM data collection and processing. Three purposes are aimed to solve, as shown in Fig. 17a, (1) in situ highresolution imaging of multiple people in one scene; (2) fast focusing on the assigned target spot with low interference; and (3) real-time monitoring on human postures and vital signs. A programmable metasurface with $32 * 24$ digital units $\left(54 * 54 \mathrm{~cm}^{2}\right)$ is realized by integrating a PIN diode on a patch structure, as shown in Fig. 17b. the related element responses are given in Fig. $17 \mathrm{c}$, in which a $180^{\circ}$ phase-difference is clearly observed. The complete system configuration is illustrated in Fig. 17b, which is divided into active and passive modes. In the active mode, two antennas act as the transmitter and receiver respectively, while in the passive mode, both of them are receivers.

The data-flow chart of the intelligent metasurface is provided in Fig. 17d, composed of metasurface manipulation and microwave data processing. The microwave data is first operated by a convolutional neural network (CNN) (IM-CNN-1) for full-body imaging. Then it is further processed by a well-developed faster CNN (Faster R-CNN) to locate the specific spot, such as chest for breath detection, and hands for gesture recognition. After the collection, the desirable coding pattern is calculated by the modified GS algorithm for the target scattering pattern. Hence the metasurface is able to reflect the energy on the specified human body part. Within this method, the interference signal is obviously eliminated, while signal noise ratio of echoes is enhanced. In addition, 


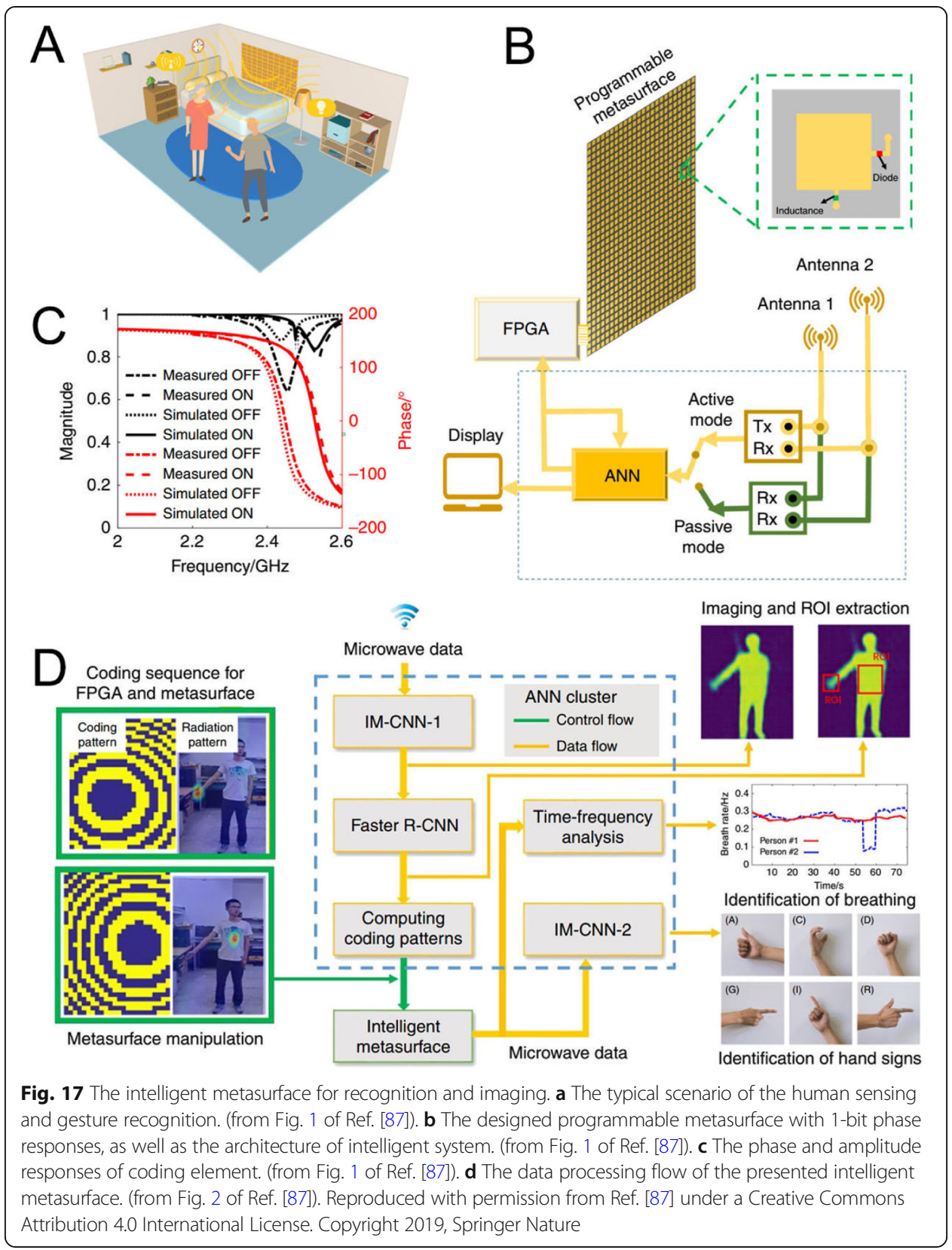

another $\mathrm{CNN}$ (IM-CNN-2) is arranged to deal with the microwave data to identify the hand signs, as listed in Fig. 17d. In the frame of the cognitive metamaterials, the establishment of recognition and learning mechanisms presented in this work may pave the farther blossom of the smart metamaterials.

\section{Conclusions}

In this paper, we review the recent five-year development of information metamaterials from the original concept to the information systems. We focus on illustrating the basic coding concept inspired by digital information theory, design methods and working mechanisms, including three important theorems. Finally, we emphatically introduce several system-level works based on the information metamaterials, from the 
programmable communication and imaging to the smart metasurfaces and recognizer. Looking back at the development trend of information metamaterials since its first presentation, we can summarize several important stages. The first stage, the initial idea of coding metamaterial was to present a new design method from digital perspective, successfully bridging the conventional physical world and digital world. This method not only simplifies the design complexity, but also exhibits superior performance on scattering pattern manipulation. The second stage, the theoretical system was further completed since the convolution operation, coding information entropy, and addition theorem were successively proposed. Besides, more functionalities like vector-beam, and more coding dimensions like polarization-bit and OAM-bit were developed during this time. The third stage, the information metamaterials evolved into programmable configuration for real-time modulations of waves and information, namely the spacetime-coding metamaterials, which dramatically promoted the progress on applications, especially on the communication and imaging systems. Several important systems were proposed on tailoring the spatial scattering fields, harmonics and the equivalent phase and amplitude responses, due to the easy reprogrammable and low-cost properties. In the fourth stage, the information metamaterials have stepped into the adaptive and smart age, with the recent works on the self-adaptively smart metasurface and intelligent imager and recognizer. An important definition of smart information metamaterials was presented, as well as a proof-of-concept device integrated with sensors and feedback channels. The smart metasurfaces can sense the surroundings and do not require human intervention anymore to decide a reaction. On the other hand, an artificial intelligence algorithm was combined for sensing and recognition on human physiological state and behaviors.

For the future stage, we envision that the information metamaterials should have cognitive ability, which necessarily includes the sensing, processing, learning, and memory abilities for the information from surroundings. In our recent works [86, 87], the primary sensing and recognition mechanisms have been explored by combining the sensors and artificial-intelligence algorithms. Upon these groundworks, higher level of integration of the metamaterial structure and system architecture deserves adequate studies. To establish the cognitive system, the present programmable metamaterials require more technical equipment including the artificial intelligence, big data, cloud processing, and so on. Except for software, the hardware framework requires evolution. On the basis of the programmable metasurfaces and FPGA controller, other parts like sensors, memory, and high-performance processors are indispensable. Moreover, all these components are better modularized, miniaturized, and highly composite, which demand long-term researches. In a word, the intelligence progress of the information metamaterials will be bewitching but challenging. With the continuous development of 5G communication networks, in the scenario of the Internet of Things and even the interconnection of everything, the application potentials of information metamaterials will fully explore. 


\section{Acknowledgements}

This work was supported by the National Key Research and Development Program of China (2017YFA0700201, 2017YFA0700202, and 2017YFA0700201), the National Natural Science Foundation of China (61631007, 61571117, 61501112, 61501117, 61522106, 61731010, 61735010, 61722106, 61701107, and 61701108), the 111 Project (111-2-05), and the Fund for International Cooperation and Exchange of National Natural Science Foundation of China (61761136007).

\section{Authors' contributions}

QM and TJC. contributed equally to this work. TJC. conceived the idea. QM. and TJC. wrote the manuscript. All authors read and approved the final manuscript.

\section{Funding}

National Key Research and Development Program of China (2017YFA0700201, 2017YFA0700202, and 2017YFA0700201):

National Natural Science Foundation of China $(61631007,61571117,61501112,61501117,61522106,61731010$,

61735010, 61722106, 61701107, and 61701108);

111 Project (111-2-05);

Fund for International Cooperation and Exchange of National Natural Science Foundation of China (61761136007).

\section{Availability of data and materials}

All data are fully available without restriction.

\section{Competing interests}

The authors declare that they have no competing interests.

Received: 2 January 2020 Accepted: 29 January 2020

Published online: 02 March 2020

\section{References}

1. Veselago VG. The Electrodynamics of Substances with Simultaneously Negative Values of $\varepsilon$ and $\mu[]]$. Sov Phys Usp. 1968 10(4):509.

2. Pendry JB, et al. Magnetism from conductors and enhanced nonlinear phenomena. 2000;47(11):2075-84.

3. Smith DR, et al. Composite medium with simultaneously negative permeability and permittivity. Phys Rev Lett. 2000; 84(18):4184-7.

4. Pendry JB. Negative refraction makes a perfect lens. Phys Rev Lett. 2000;85(18):3966-9.

5. Eleftheriades GV, Iyer AK, Kremer PC. Planar negative refractive index media using periodically LC loaded transmission lines. IEEE Trans Microw Theory Tech. 2002;50(12):2702-12.

6. Hoffman AJ, et al. Negative refraction in semiconductor metamaterials. Nat Mater. 2007:6(12):946-50

7. Houck AA, et al. Experimental observations of a left-handed material that obeys Snell's law. Phys Rev Lett. 2003;90(13): 137401

8. Shelby RA, et al. Experimental verification of a negative index of refraction. Science. 2001;292(5514):77-9.

9. Mocella $\mathrm{V}$, et al. Self-collimation of light over millimeter-scale distance in a quasi-zero-average-index metamaterial. Phys Rev Lett. 2009;102(13):133902

10. Huang $X$, et al. Dirac cones induced by accidental degeneracy in photonic crystals and zero-refractive-index materials. Nat Mater. 2011;10(8):582

11. Feng S. Loss-induced omnidirectional bending to the normal in -near-zero metamaterials. Phys Rev Lett. 2012;108(19): 193904.

12. Schurig D, et al. Metamaterial electromagnetic cloak at microwave frequencies. Science. 2006;314(5801):977-80.

13. Kundtz N, Smith DR. Extreme-angle broadband metamaterial lens. Nat Mater. 2010:9(2):129.

14. Ergin T, et al. Three-dimensional invisibility cloak at optical wavelengths. Science. 2010;328(5976):337-9.

15. Hui FM, Cui TJ. Three-dimensional broadband ground-plane cloak made of metamaterials. Nat Commun. 2010;1(3):21.

16. $\mathrm{Ma} \mathrm{Q}$, et al. Experiments on active cloaking and illusion for Laplace equation. Phys Rev Lett. 2013;111(17):173901.

17. Yang F, et al. DC electric invisibility cloak. Phys Rev Lett. 2012;109(5):053902.

18. Cheng $\mathrm{Q}$, et al. An omnidirectional electromagnetic absorber made of metamaterials. New J Physics. 12(6):063006

19. Sheng C, et al. Trapping light by mimicking gravitational lensing. Nat Photonics. 2013;7(11):902.

20. Ramakrishna SA, et al. Imaging the near field. J Mod Opt. 2003;50(9):1419-30.

21. Jiang WX, et al. Broadband All-Dielectric Magnifying Lens for Far-Field High-Resolution Imaging. Adv Mater. 25(48):6963-8.

22. Xiang W, et al. A broadband transformation-optics metasurface lens. Applied Physics Lett. 2014;104(15):151601-4.

23. $\mathrm{Ma} \mathrm{Q}$, et al. Broadband metamaterial lens antennas with special properties by controlling both refractive-index distribution and feed directivity. J Opt. 2018;20(4):045101.

24. Mei ZL, et al. A half Maxwell fish-eye lens antenna based on gradient-index metamaterials. IEEE Trans Antennas Propag. 2011:60(1):398-401.

25. Qi MQ, et al. Tailoring radiation patterns in broadband with controllable aperture field using metamaterials. IEEE Trans Antennas Propag. 2013;61(11):5792-8.

26. Chen $\mathrm{X}$, et al. Three-dimensional broadband and high-directivity lens antenna made of metamaterials. J Appl Physics. 110(4):044904.

27. Lin $\mathrm{XQ}$, et al. Controlling electromagnetic waves using tunable gradient dielectric metamaterial lens. Appl Phys Lett. 92(13):131904.

28. Holloway CL, Dienstfrey A, Kuester EF, O'Hara JF, Azad AK, Taylor AJ. Metamaterials. 2009:3:100.

29. Akselrod GM, et al. Large-area metasurface perfect absorbers from visible to near-infrared. Adv Mater. 2015;27(48):8028-34. 
30. Yao Y, et al. Electrically tunable metasurface perfect absorbers for ultrathin mid-infrared optical modulators. Nano Lett. 2014;14(11):6526-32.

31. Li J, et al. Bidirectional perfect absorber using free substrate plasmonic metasurfaces. Adv Optical Mater. 2017;5(12): 1700152.

32. Guo W, et al. Ultra-broadband infrared metasurface absorber. Opt Express. 2016;24(18):20586-92.

33. Bossard JA, et al. Near-ideal optical metamaterial absorbers with super-octave bandwidth. ACS Nano. 2014;8(2):1517-24.

34. Ding $X$, et al. Ultrathin Pancharatnam-berry metasurface with maximal cross-polarization efficiency. Adv Mater. 2015; 27(7):1195-200.

35. Cong L, et al. A perfect metamaterial polarization rotator. Appl Phys Lett. 2013;103(17):171107.

36. Su P, et al. An ultra-wideband and polarization-independent Metasurface for RCS reduction. Sci Rep. 2016;6:20387.

37. Cheng YZ, et al. Ultrabroadband reflective polarization convertor for terahertz waves. Appl Phys Lett. 2014;105(18):26.

38. Pfeiffer C, Grbic A. Controlling vector Bessel beams with metasurfaces. Phys Rev Appl. 2014;2(4):044012.

39. Yin $X$, et al. Photonic spin hall effect at metasurfaces. Science. 2013;339(6126):1405-7.

40. Lin J, et al. Nanostructured holograms for broadband manipulation of vector beams. Nano Lett. 2013;13(9):4269-74.

41. Yu P, et al. Generation of vector beams with arbitrary spatial variation of phase and linear polarization using plasmonic metasurfaces. Opt Lett. 2015;40(14):3229-32.

42. Yu N, Capasso F. Flat optics with designer metasurfaces. Nat Mater. 2014;13(2):139-50.

43. Huang $L$, et al. Broadband hybrid holographic multiplexing with geometric metasurfaces. Adv Mater. 2015;27(41):6444-9.

44. Zheng G, et al. Metasurface holograms reaching 80\% efficiency. Nat Nanotechnol. 2015;10(4):308.

45. Shalaev Ml, et al. High-efficiency all-dielectric metasurfaces for ultracompact beam manipulation in transmission mode. Nano Lett. 2015;15(9):6261-6.

46. Zhang $\mathrm{H}$, et al. High-efficiency dielectric metasurfaces for polarization-dependent terahertz wavefront manipulation. Adv Optical Mater. 2018;6(1):1700773.

47. Luo J, et al. Highly efficient wavefront manipulation in terahertz based on plasmonic gradient metasurfaces. Opt Lett. 2014;39(8):2229-31.

48. Cheng J, et al. Wave manipulation with designer dielectric metasurfaces. Opt Lett. 2014:39(21):6285-8.

49. Liu X, et al. Experimental realization of a terahertz all-dielectric metasurface absorber. Opt Express. 2017;25(1):191-201.

50. Ma G, et al. Acoustic metasurface with hybrid resonances. Nat Mater. 2014;13(9):873.

51. Xie B, et al. Coding acoustic metasurfaces. Adv Mater. 2017;29(6):1603507.

52. Xie $Y$, et al. Wavefront modulation and subwavelength diffractive acoustics with an acoustic metasurface. Nat Commun. 2014;5:5553.

53. Mei J, Wu Y. Controllable transmission and total reflection through an impedance-matched acoustic metasurface. New J Phys. 2014;16(12):123007.

54. Esfahlani H, et al. Acoustic carpet cloak based on an ultrathin metasurface. Phys Rev B. 2016;94(1):014302.

55. Zhao J, et al. Achieving flexible low-scattering metasurface based on randomly distribution of meta-elements. Opt Express. 2016;24(24):27849-57.

56. Cui TJ, et al. Coding metamaterials, digital metamaterials and programmable metamaterials. Light Sci Appl. 2014;3:e218.

57. Cui TJ. Microwave metamaterials. Natl Sci Rev. 2018;5(2):134-6.

58. Liu S, et al. Convolution operations on coding Metasurface to reach flexible and continuous controls of terahertz. Adv Sci. 2016;3(10):1600156.

59. Cui T-J, et al. Information entropy of coding metasurface. Light Sci Appl. 2016;5:e16172.

60. Wu RY, et al. Addition theorem for digital coding Metamaterials. Adv Optical Mater. 2018;6(5).

61. Li J, et al. Amplitude modulation of anomalously reflected terahertz beams using all-optical active Pancharatnam-berry coding metasurfaces. Nanoscale. 2019;11(12):5746-53.

62. Bao $L$, et al. Design of digital coding metasurfaces with independent controls of phase and amplitude responses. Appl Phys Lett. 2018;113(6):063502.

63. Wu RY, et al. Digital Metasurface with phase code and reflection-transmission amplitude code for flexible full-space electromagnetic manipulations. Adv Optical Mater. 2019;7(8):1801429.

64. Ma Q, et al. Beam-editing coding Metasurfaces based on polarization bit and orbital-angular-momentum-mode bit. Adv Optical Mater. 2017:5(23):1700548.

65. Sui S, et al. Symmetry-based coding method and synthesis topology optimization design of ultra-wideband polarization conversion metasurfaces. Appl Phys Lett. 2016;109(1):014104.

66. Iqhal S, et al. Polarization-selective dual-band digits coding metasurface for controls of transmitted waves. J Physics DAppl Physics. 2018;51(28):285103.

67. Ding $\mathrm{G}$, et al. Dual-Helicity decoupled coding Metasurface for independent spin-to-orbital angular momentum conversion. Phys Rev Appl. 2019;11(4):044043.

68. Han J, et al. 1-bit digital orbital angular momentum vortex beam generator based on a coding reflective metasurface. Optical Materials Express. 2018:8(11):3470-8.

69. Zheng $\mathrm{Q}$, et al. Efficient orbital angular momentum vortex beam generation by generalized coding metasurface. Appl Phys. 2019;125(2):136.

70. Ji W, et al. High-efficiency and ultra-broadband asymmetric transmission metasurface based on topologically coding optimization method. Opt Express. 2019;27(3):2844-54.

71. Katare KK, et al. Realization of Split beam antenna using transmission-type coding Metasurface and planar Lens. IEEE Trans Antennas Propag. 2019;67(4):2074-84.

72. Li F-F, et al. Transmission and radar cross-section reduction by combining binary coding metasurface and frequency selective surface. Opt Express. 2018;26(26):33878-87.

73. Liu S, et al. Anomalous refraction and nondiffractive Bessel-beam generation of terahertz waves through transmissiontype coding Metasurfaces. Acs Photonics. 2016:3(10):1968-77.

74. Shen Z, et al. Design of transmission-type coding metasurface and its application of beam forming. Appl Phys Lett. 2016;109(12):121103. 
75. Zhang L, et al. Transmission-reflection-integrated multifunctional coding Metasurface for full-space controls of electromagnetic waves. Adv Funct Mater. 2018;28(33):1802205.

76. Wan X, et al. Field-programmable beam reconfiguring based on digitally-controlled coding metasurface. Sci Rep. 2016;6: 20663.

77. Zhang L, et al. Space-time-coding digital metasurfaces. Nat Commun. 2018;9(1):4334.

78. Dai JY, et al. Independent control of harmonic amplitudes and phases via a time-domain digital coding metasurface. Light Sci Appl. 2018;7:90.

79. Cui TJ, et al. Direct transmission of digital message via programmable coding metasurface. Research. 2019;2019:2584509.

80. Zhao J, et al. Programmable time-domain digital-coding metasurface for non-linear harmonic manipulation and new wireless communication systems. Natl Sci Rev. 2019;6(2):231-8.

81. Li L, et al. Electromagnetic reprogrammable coding-metasurface holograms. Nat Commun. 2017;8:197.

82. Li L, et al. Machine-learning reprogrammable metasurface imager. Nat Commun. 2019;10:1082.

83. Li YB, et al. Transmission-type 2-bit programmable Metasurface for single-sensor and single-frequency microwave imaging. Sci Rep. 2016;6.

84. $\mathrm{Ma} \mathrm{Q}$, et al. Controllable and programmable nonreciprocity based on detachable digital coding Metasurface. Adv Optical Mater. 2019;7:1901285.

85. Zhang $L$, et al. Breaking reciprocity with space-time-coding digital Metasurfaces. Adv Mater. 2019;31(41):1904069.

86. $\mathrm{Ma} \mathrm{Q}$, et al. Smart metasurface with self-adaptively reprogrammable functions. Light Sci Appl. 2019;8(1):1-12.

87. Li L, et al. Intelligent metasurface imager and recognizer. Light Sci Appl. 2019;8(1):1-9.

88. Gao L-H, et al. Broadband diffusion of terahertz waves by multi-bit coding metasurfaces. Light Sci Appl. 2015;4:e324.

89. Zhang XG, et al. Light-controllable digital coding Metasurfaces. Advanced Sci. 2018;5(11):1801028.

90. Liu S, et al. Anisotropic coding metamaterials and their powerful manipulation of differently polarized terahertz waves. Light Sci Appl. 2016;5:e16076.

91. Yu NF, et al. Light propagation with phase discontinuities: generalized Laws of reflection and refraction. Science. 2011; 334(6054):333-7.

92. Chen K, Zhang N, Ding G, Zhao J, Jiang T, Feng Y. Active Anisotropic Coding Metasurface with Independent Real-Time Reconfigurability for Dual Polarized Waves. Advanced Materials Technologies. 2019;19:1900930.

93. Tan H, Deng J, Zhao R, Wu X, Li G, Huang L, Reviews P. A free-space orbital angular momentum multiplexing communication system based on a Metasurface. Laser Photonics Rev. 2019;13(6):1800278.

94. Zhang $L$, et al. Spin-controlled multiple pencil beams and vortex beams with different polarizations generated by Pancharatnam-berry coding Metasurfaces. ACS Appl Mater Interfaces. 2017;9(41):36447-55.

95. Shannon CE. A mathematical theory of communication. Bell Syst Tech J. 1948;27:379-423.

96. Shcherbakov MR, Lemasters R, Fan Z, Song J, Lian T, Harutyunyan H, Shvets GJO. Time-variant metasurfaces enable tunable spectral bands of negative extinction. Optica. 2019;6(11):1441-2.

97. Guo X, Ding Y, Duan Y, Ni X. Nonreciprocal Metasurface with space-time phase modulation. Light Sci Appl. 2019;8:1-9.

98. Tse D. Fundamentals of wireless communication: Cambridge University Press; 2004.

99. Hunt J, Driscoll T, Mrozack A. Metamaterial Apertures for Computational Imaging. Science. 339(6117):310-3.

100. Xu X, Peng B, Li D. Flexible Visible-Infrared Metamaterials and Their Applications in Highly Sensitive Chemical and Biological Sensing. Nano Lett. 11(8):3232-8.

101. Li Z, Zhang T, Wang Y, Kong W, Zhang J, Huang Y, Reviews P. Achromatic broadband super-resolution imaging by super-oscillatory metasurface. Laser Photonics Rev. 2018;12(10):1800064.

102. Chen K, Ding G, Hu G, Jin Z, Zhao J, Feng Y, Qiu CWJAM. Directional Janus Metasurface. Adv Mater. 2019;32:e1906352.

103. Duarte MF, et al. Single-pixel imaging via compressive sampling. IEEE Signal Process Mag. 2008;25:83.

104. Zhao W, et al. Full-color hologram using spatial multiplexing of dielectric metasurface. Opt Lett. 2016;41:147.

105. Ghanekar A, et al. High-rectification near-field thermal diode using phase lichange periodic nanostructure. Appl Phys Lett. 2016;109:123106.

106. Wang L, Li L, Li Y, Zhang H, Cui TJ. Single-shot and single-sensor high/super-resolution microwave imaging based on metasurface. Sci Rep. 2016;6:26959.

107. Fromenteze T, et al. Computational polarimetric microwave imaging. Opt Express. 2017;25:27488-505.

108. Neifeld MA, Shankar P. Feature-specific imaging. Appl Opt. 2003:42:3379-89.

109. Pal HS, Ganotra D, Neifeld MA. Face recognition by using featurespecific imaging. Appl Opt. 2005;44:3378-794.

110. Nayar SK, Branzoi V. Programmable imaging: toward a flexible camera. Int J Comput Vis. 2006;20:7-22.

111. Kulkarni K, Turaga P. Reconstruction-free action inference from compressive imagers. IEEE Trans Pattern Anal Mach Intell. 2016;38:772-84.

112. Liu S, Cui TJ. Concepts, working principles, and applications of coding and programmable Metamaterials. Adv Opt Mater. 2017;5(22):1700624.

113. Cui TJ, et al. Information metamaterials and metasurfaces. J Mater Chem C. 2017;5(15):3644-68.

114. Xiao J, et al. A survey on wireless indoor localization from the device perspective. ACM Comput Surv. 2016;49:25.

115. Pu QF, et al. Whole-home gesture recognition using wireless signals. ACM, Miami, Florida: Proceedings of the 19th Annual International Conference on Mobile Computing \& Networking; 2013. p. 27-38.

116. Sadreazami H, et al. CapsFall: fall detection using ultra-wideband radar and capsule network. IEEE Access. 2019;7:55336-43.

117. Zhao MM, et al. Through-wall human pose estimation using radio signals. IEEE, Salt Lake City, UT: Proceedings of 2018 IEEE/CVF Conference on Computer Vision and Pattern Recognition; 2018. p. 7356-65.

\section{Publisher's Note}

Springer Nature remains neutral with regard to jurisdictional claims in published maps and institutional affiliations. 